JOURNAL OF

SYMPLECTIC GEOMETRY

Volume 1, Number 1, 1-46, 2001

\title{
The Verlinde formulas as fixed point formulas
}

\author{
A. Alekseev, E. Meinrenken and C. Woodward
}

We express the index of the $\operatorname{Spin}_{c}$-Dirac operator on symplectic quotients of a Hamiltonian loop group manifold in terms of fixed point data. As an application we prove the Verlinde formulas for the $\operatorname{Spin}_{c}$-quantization of moduli spaces of flat bundles over surfaces.

\section{Introduction.}

The goal of this paper is to show that the Verlinde formulas [38] for the geometric quantization of moduli spaces of flat bundles over surfaces follow from a fixed point principle for loop group actions, in much the same way as the Weyl or Steinberg formulas can be interpreted as fixed point formulas for flag varieties [5].

Our fixed point formula is a loop group version of the following principle for actions of compact, connected groups $G$. Let $M$ be a compact Hamiltonian $G$-manifold with $G$-equivariant pre-quantum line bundle $L$. Let $\chi(M)$ be the $G$-equivariant index of the $\operatorname{Spin}_{c}$-Dirac operator with values in $L$. The virtual character $\chi(M)$ can be computed from the Atiyah-Segal-Singer theorem in terms of fixed point data, or from the quantization commutes with reduction principle in terms of indices of symplectic quotients. A combination of these two expressions leads to formulas for indices of symplectic quotients as a sum of fixed point contributions. For instance, the index of a symplectic quotient of a coadjoint orbit by the action of a maximal torus is given by the Weyl character formula, which is a sum over fixed points of the maximal torus, indexed by elements of the Weyl group.

Our main result is a similar fixed point formula for pre-quantized Hamiltonian $L G$-manifolds $\widehat{M}$ with proper moment map, for $G$ simply connected. While $\widehat{M}$ itself is infinite-dimensional, the properness assumption implies that its symplectic quotients are compact. To define fixed point contributions we consider a finite dimensional compact $G$-manifold $M$, obtained from $\widehat{M}$ as a quotient by the based loop group $\Omega G \subset L G$. Theorem 4.3 below expresses the indices of symplectic quotients of $\widehat{M}$ in terms of fixed point data on $M$. The fixed point contributions are reminiscent of the right hand side 
of the equivariant index theorem, although $M$ does not carry a naturally

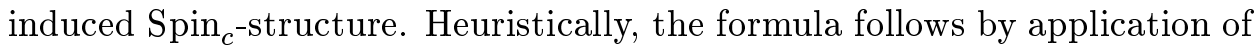
the equivariant index theorem to the loop group manifold, and subsequent "renormalization" of the infinities on both sides.

When we apply the formula to the action of the loop group on the moduli space of flat bundles on a Riemann surface, and combine our formula with recent results of Teleman [34] on vanishing of the higher cohomology, we obtain a short proof of the Verlinde formula. It differs from the original proof given by Tsuchiya-Ueno-Yamada [36], who used the degeneration of the surface and a computation of the Verlinde numbers for the three-holed sphere. Another proof of the Verlinde formula that does not use degeneration was outlined by Szenes in [33], and carried out for $\mathrm{SU}(2)$ and $\mathrm{SU}(3)$. The proof was extended by Jeffrey-Kirwan [21] to $\mathrm{SU}(n)$, and Bismut-Labourie [10] to arbitrary compact, connected simply-connected groups, for sufficiently high level. The idea of deriving the Verlinde formula from localization appears in the physics papers by Gerasimov [18] and Blau and Thompson [11]. The problem of finding a rigorous approach via localization had been suggested to us by A. Szenes many years ago. Partial results in this direction were obtained by S. Chang [13].

An interesting outstanding question is whether our formula may be fit into the framework of twisted K-theory, as applied to the Verlinde algebra by Freed, Hopkins, and Teleman [16].

The contents of the paper are as follows. In Section 2 we discuss Spin $_{c^{-}}$ quantization of finite dimensional Hamiltonian manifolds, and describe the finite dimensional version of our fixed point formula. Section 3 is dedicated to a review of loop group actions and group-valued moment maps. The main theorem is stated in Section 4. In Section 5 we apply the theorem to a calculation of Verlinde numbers, and in Section 6 we describe its proof.

\section{Notation.}

Throughout the paper $G$ will denote a compact, connected Lie group, and $\mathfrak{g}$ its Lie algebra. We denote by $R(G)$ the ring of characters of finitedimensional virtual representations. We let $T$ be a maximal torus in $G$, and $\mathfrak{t}$ its Lie algebra. The integral lattice $\Lambda \subset \mathfrak{t}$ is defined as the kernel of the exponential map exp $: \mathfrak{t} \rightarrow T$, and the (real) weight lattice $\Lambda^{*} \subset \mathfrak{t}^{*}$ is its dual. Embed $\mathfrak{t}^{*} \hookrightarrow \mathfrak{g}^{*}$ as the fixed point set for the coadjoint action of $T$. Every $\mu \in \Lambda^{*}$ defines a 1 -dimensional $T$-representation, denoted $\mathbb{C}_{\mu}$, where $t=\exp \xi$ acts by $t^{\mu}:=e^{2 \pi i\langle\mu, \xi\rangle}$. This representation extends uniquely to the stabilizer group $G_{\mu}$ of $\mu$. We let $W$ be the Weyl group of $(G, T)$ and $\Re \subset \Lambda^{*}$ 
the set of roots. We fix a set of positive roots $\Re_{+} \subset \mathfrak{R}$ and let $\mathfrak{t}_{+} \subset \mathfrak{t}$ and $\mathfrak{t}_{+}^{*} \subset \mathfrak{t}^{*}$ be the corresponding positive Weyl chambers. For any dominant weight $\mu \in \Lambda_{+}^{*}:=\Lambda^{*} \cap \mathfrak{t}_{+}^{*}$ we denote by $V_{\mu}$ the irreducible representation with highest weight $\mu$ and by $\chi_{\mu} \in R(G)$ its character. Some additional notation to be introduced later:

$\begin{array}{ll}J & \text { Weyl denominator; } 2.4 \\ \mathfrak{A} & \text { fundamental alcove; } 2.4 \\ \rho, \alpha_{0}, c & \text { half-sum of positive roots, highest root, } \\ & \text { dual Coxeter number; } 2.4 \\ B=B_{k} & \text { inner product on } \mathfrak{g}, 2.4 \\ \Lambda_{k}^{*}, R_{k}(G) & \text { level } k \text { weights, level } k \text { characters; } 2.4 \\ T_{k} & \text { a certain finite subgroup of } T ; 2.4 \\ t_{\lambda} & \text { element of } T_{k+c} \text { parametrized by } \lambda \in \Lambda_{k}^{*} ; 2.4 \\ G_{\sigma}, L G_{\sigma} & \text { stabilizer in } G \text { resp. } L G \text { of face } \sigma \subset \mathfrak{A} ; 3.1 \\ \mathfrak{R}_{+, \sigma}, \rho_{\sigma}, \mathfrak{t}_{+, \sigma} & \text { positive roots for } G_{\sigma}, \text { their half-sum, } \\ & \text { positive Weyl chamber; 3.1 } \\ \gamma_{\sigma} & \text { distinguished point in face } \sigma \subset \mathfrak{A} ; 3.1 \\ L G, \Omega G & \text { free loop group, based loop group; } 3.1 \\ W_{\text {aff }} & \text { affine Weyl group; } 3.1 \\ \widehat{L G}(k) & \text { level } k \text { central extension of the loop group; } 4.1 \\ \theta^{L}, \theta^{R} & \text { left, right Maurer-Cartan forms; } 3.1\end{array}$

2. $\operatorname{Spin}_{\mathrm{c}}$-quantization of symplectic manifolds.

In this section we review $\operatorname{Spin}_{c}$-quantization for compact Hamiltonian Gmanifolds. We explain how the fixed point formula for the equivariant index, together with the "quantization commutes with reduction" principle, leads to a formula (7) for the index of a symplectic quotient in terms of fixed point contributions for a certain finite subgroup. This is the finite-dimensional version of the main result of the paper.

\section{1. $\operatorname{Spin}_{c}$-quantization of Hamiltonian $G$-spaces.}

We refer to Lawson-Michelson [24] for background on Spin $_{c}$-structures, and to Duistermaat [15] for a discussion of the symplectic case. 
Let $M$ be a compact, connected manifold with symplectic form $\omega$, together with a symplectic $G$-action. Given $\xi \in \mathfrak{g}$ let $\xi_{M}=\left.\frac{\partial}{\partial t}\right|_{t=0} \exp (t \xi)^{*}$ denote the corresponding vector field on $M$. The action is called Hamiltonian if there exists a $G$-equivariant map $\Phi \in C^{\infty}\left(M, \mathfrak{g}^{*}\right)^{G}$ such that $\iota\left(\xi_{M}\right) \omega=\mathrm{d} \Phi(\xi)$ for all $\xi$. The map $\Phi$ is called a moment map and the triple $(M, \omega, \Phi)$ is called a Hamiltonian $G$-space. By a theorem of Kirwan [22], the intersection $\Phi(M) \cap \mathfrak{t}_{+}^{*}$ is a convex polytope; it is called the moment polytope of $(M, \omega, \Phi)$.

Suppose $(M, \omega, \Phi)$ carries a $G$-equivariant pre-quantum line bundle $L$. That is, $L$ comes equipped with an invariant connection $\nabla$, such that the Chern form $c_{1}(L)=\frac{i}{2 \pi} \operatorname{curv}(\nabla)$ is equal to $\omega$ and the vertical part of the vector field $\xi_{L}$ is given by Kostant's formula [23]

$$
\operatorname{Vert}\left(\xi_{L}\right)=2 \pi \Phi(\xi) \frac{\partial}{\partial \phi}
$$

where $\frac{\partial}{\partial \phi}$ is the generator for the scalar $S^{1}$-action on the fibers of $L$. Choose an invariant almost complex structure $I$ on $M$ that is compatible with $\omega$, in the sense that $\omega(\cdot, I \cdot)$ defines a Riemannian metric. The almost complex structure $I$ defines a $G$-equivariant $\operatorname{Spin}_{c}$-structure on $M$, which we twist by the line bundle $L$. Any choice of Hermitian connection on $T M$ defines a Dirac operator $\not \partial$ for the twisted $\operatorname{Spin}_{c}$-structure, and we define $\chi(M) \in R(G)$ as its equivariant index

$$
\chi(M)=\operatorname{index}_{G}(\not \partial) \in R(G) .
$$

The index is independent of the choice of $I$ and of the connection. In case $M$ is Kähler and $L$ is holomorphic, the index coincides with the Euler characteristic for the sheaf of holomorphic sections of $L$.

\subsection{Quantization commutes with reduction.}

Following [27] we call a point $\mu \in \mathfrak{g}^{*}$ a quasi-regular value of $\Phi$ if all $G_{\mu^{-}}$ orbits in $\Phi^{-1}(\mu)$ have the same dimension. This includes regular values and weakly regular values of $\Phi$. For any quasi-regular value $\mu \in \mathfrak{g}^{*}$ the reduced space (symplectic quotient) $M_{\mu}=\Phi^{-1}(\mu) / G_{\mu}$ is a symplectic orbifold. If one drops the quasi-regularity assumption, the space $M_{\mu}$ acquires more serious singularities (cf. [32]). For any dominant weight $\mu \in \Lambda_{+}^{*}$ which is a quasiregular value of $\Phi$,

$$
L_{\mu}:=\left(\left.L\right|_{\Phi^{-1}(\mu)} \otimes \mathbb{C}_{-\mu}\right) / G_{\mu} \rightarrow M_{\mu}
$$


is a pre-quantum orbifold-line bundle over $M_{\mu}$. The definition of $\operatorname{Spin}_{c^{-}}$ index carries over to the orbifold case, hence $\chi\left(M_{\mu}\right)$ is defined. In [27], this is extended further to the case of singular symplectic quotients, using a partial desingularization. The following Theorem was conjectured by GuilleminSternberg and is known as "quantization commutes with reduction".

Theorem 2.1 ([26, 27]). Let $(M, \omega, \Phi)$ be a compact pre-quantized Hamiltonian $G$-manifold. Then the multiplicity of $\chi_{\mu}$ in $\chi(M) \in R(G)$ is equal to $\chi\left(M_{\mu}\right)$.

In particular, only weights $\mu \in \Lambda_{+}^{*}$ which are contained in the moment polytope $\Phi(M) \cap \mathfrak{t}_{+}^{*}$ can appear in $\chi(M)$.

\subsection{Equivariant index theorem.}

The equivariant index theorem expresses the value $\chi(M, g)$ in terms of local data at the fixed point set $M^{g}$. We recall that the connected components $F \subseteq M^{g}$ are compact, embedded almost complex submanifolds of $M$. They are invariant under the action of the centralizer $G_{g}$, and the pull-backs $\omega_{F}, \Phi_{F}, L_{F}$ of $\omega, \Phi, L$ give $F$ the structure of a pre-quantized Hamiltonian $G_{g^{-}}$-space. The action of $g \in G$ on $L$ restricts to a multiplication by a phase factor $\mu_{F}(g) \in \mathrm{U}(1)$ on $L_{F}$.

Let $\operatorname{Td}(F)$ be the Todd form, for any Hermitian connection on $T F$, and let the form $\mathcal{D}_{\mathbb{C}}\left(\nu_{F}, g\right)$ be defined by

$$
\mathcal{D}_{\mathbb{C}}\left(\nu_{F}, g\right)=\operatorname{det}_{\mathbb{C}}\left(1-A_{F}(g)^{-1} e^{R_{\nu_{F}} / 2 \pi}\right) .
$$

Here $A_{F}(g) \in \Gamma^{\infty}\left(\mathrm{U}\left(\nu_{F}\right)\right)$ is the unitary bundle automorphism of $\nu_{F}$ induced by $g$, and $R_{\nu_{F}} \in \Omega^{2}\left(F, \mathfrak{u}\left(\nu_{F}\right)\right)$ the curvature of an invariant Hermitian connection on $\nu_{F}$. The Atiyah-Segal-Singer fixed point formula [7,6] asserts that

$$
\chi(M, g)=\sum_{F \subseteq M^{g}} \chi\left(\nu_{F}, g\right)
$$

where

$$
\chi\left(\nu_{F}, g\right)=\mu_{F}(g) \int_{F} \frac{\operatorname{Td}(F) e^{c_{1}\left(\left.L\right|_{F}\right)}}{\mathcal{D}_{\mathbb{C}}\left(\nu_{F}, g\right)} .
$$

(For finite fixed point set, the formula is a special case of the Atiyah-Bott Lefschetz formula [4].) We will also need an equivalent expression for the 
fixed point contributions, in which the almost complex structure enters only via the $\operatorname{Spin}_{c}$-line bundle $\mathcal{L}$, given as a tensor product $\mathcal{L}=L^{2} \otimes K^{-1}$ of $L \otimes L$ with the anti-canonical line bundle $K^{-1}$ for the almost complex structure:

$$
\chi\left(\nu_{F}, g\right)=\zeta_{F}(g)^{1 / 2} \int_{F} \frac{\hat{A}(F) e^{\frac{1}{2} c_{1}\left(\left.\mathcal{L}\right|_{F}\right)}}{\mathcal{D}_{\mathbb{R}}\left(\nu_{F}, g\right)} .
$$

Here $\zeta_{F}(g)$ is the eigenvalue for the action of $g$ on $\left.\mathcal{L}\right|_{F}$, and the square root $\zeta_{F}(g)^{1 / 2}=\mu_{F}(g) \kappa_{F}(g)^{-1 / 2}$ is defined as

$$
\kappa_{F}(g)^{-1 / 2}:=\operatorname{det}\left(A_{F}(g)^{1 / 2}\right) .
$$

where $A_{F}(g)^{1 / 2} \in \Gamma^{\infty}\left(U\left(\nu_{F}\right)\right)$ is the unique square root of $A_{F}(g)$ having all its eigenvalues in the set $\left\{e^{i \phi} \mid 0 \leq \phi<\pi\right\}$. Furthermore $\widehat{A}(F)$ is the $\hat{A}$-form of $F$, and

$$
\mathcal{D}_{\mathbb{R}}\left(\nu_{F}, g\right)=i^{\operatorname{rank}\left(\nu_{F}\right) / 2} \operatorname{det}_{\mathbb{R}}^{1 / 2}\left(1-A_{F}(g)^{-1} e^{R_{\nu_{F}} / 2 \pi}\right),
$$

viewing $A_{F}(g)$ as a real automorphism, $R_{\nu_{F}}$ as a $\mathfrak{o}\left(\nu_{F}\right)$-valued 2-form, and taking the positive square root. The fixed point expressions (3) and (4) are identical because

$$
\mathcal{D}_{\mathbb{C}}\left(\nu_{F}, g\right)=\mathcal{D}_{\mathbb{R}}\left(\nu_{F}, g\right) e^{\frac{1}{2} c_{1}\left(K_{\nu_{F}}\right)} \kappa_{F}(g)^{1 / 2}
$$

and

$$
\operatorname{Td}(F)=\hat{A}(F) e^{-\frac{1}{2} c_{1}\left(K_{F}\right)}
$$

where $K_{F}$ is the canonical bundle of $F$ and $K_{\nu_{F}}$ that for $\nu_{F}$.

\subsection{Fixed point formula for multiplicities.}

We now use finite Fourier transform to extract the multiplicity of any weight $\mu \in \Lambda_{+}^{*}$ from Formula (2). For a different approach using Fourier series, see Guillemin-Prato [19]. For simplicity, we only consider the case where $G$ is simply connected.

We need to introduce some extra notation and facts regarding compact Lie groups. Suppose (for a short moment) that $G$ is simple. Let $\alpha_{0} \in \Lambda^{*}$ be the highest root, $h_{\alpha_{0}} \in \mathfrak{t}$ its coroot, $\rho \in \Lambda^{*}$ the half-sum of positive roots, and $c=1+\rho\left(h_{\alpha_{0}}\right)$ the dual Coxeter number. The fundamental alcove is denoted $\mathfrak{A}:=\left\{\xi \in \mathfrak{t}_{+} \mid \alpha_{0}(\xi) \leq 1\right\}$. Let the basic inner product $B^{G}$ be the unique invariant inner product on $\mathfrak{g}$ such that $B^{G}\left(h_{\alpha_{0}}, h_{\alpha_{0}}\right)=2$. It has the 
important property that it restricts to an integer-valued $\mathbb{Z}$-bilinear form on the lattice $\Lambda$ (see [12], Chapter V.2).

For a general simply connected group $G$, decompose into simple factors $G=G_{1} \times \ldots \times G_{s}$ with dual Coxeter numbers $c=\left(c_{1}, \ldots, c_{s}\right)$, and define $\mathfrak{A}=\mathfrak{A}_{1} \times \ldots \times \mathfrak{A}_{s}$. Any invariant symmetric bilinear form on $\mathfrak{g}$ can be written

$$
B_{k}:=\sum_{j=1}^{s} k_{j} B^{G_{j}},
$$

where $k_{j} \in \mathbb{R}$. We denote by $B_{k}^{b}: \mathfrak{g} \rightarrow \mathfrak{g}^{*}$ the linear map defined by $B_{k}$, and if all $k_{j} \neq 0$ the inverse map is denoted $B_{k}^{\sharp}=\left(B_{k}^{b}\right)^{-1}$. Suppose all $k_{j}$ are positive integers. Then $B_{k}$ is integer-valued on $\Lambda$, and we have inclusions $B_{k}^{b}(\Lambda) \subset \Lambda^{*}$ and $B_{k}^{\sharp}\left(\Lambda^{*}\right) \supset \Lambda$. The finite Fourier transform is taken using the finite subgroup

$$
T_{k}:=B_{k}^{\sharp}\left(\Lambda^{*}\right) / \Lambda
$$

of $T=\mathfrak{t} / \Lambda$. Let

$$
\mathfrak{A}_{k}^{*}:=B_{k}^{b}(\mathfrak{A}) \subset \mathfrak{t}_{+}^{*}, \quad \Lambda_{k}^{*}=\Lambda^{*} \cap \mathfrak{A}_{k}^{*} .
$$

The weights in $\Lambda_{k}^{*}$ are called weights at level $k$. Using the definition of the alcove, one verifies that the map

$$
\Lambda_{k}^{*} \rightarrow T_{k+c}, \lambda \mapsto t_{\lambda}:=\exp \left(B_{k+c}^{\sharp}(\lambda+\rho)\right)
$$

takes values in $T_{k+c}^{\mathrm{reg}}=T_{k+c} \cap G^{\mathrm{reg}}$ and identifies $\Lambda_{k}^{*}=T_{k+c}^{\mathrm{reg}} / W$.

Example 2.2. Suppose $G=\mathrm{SU}(2)$. Then $\rho \in \mathfrak{t}^{*}$ spans the weight lattice, and $\alpha_{0}=2 \rho$ is the positive root. Therefore, $B_{k}^{b}(\Lambda)=2 k \Lambda^{*}$ and $T_{k}=\mathbb{Z}_{2 k}$. Furthermore, $\mathfrak{A}_{k}^{*}=\{t \rho \mid 0 \leq t \leq k\}$ and $\Lambda_{k}^{*}=\{0, \rho, \ldots, k \rho\}$. The dual Coxeter number is $c=2$. For $\lambda=l \rho$, the element $t_{\lambda}$ is a diagonal matrix with entries $e^{i \phi}, e^{-i \phi}$ down the diagonal, where $\phi=\pi \frac{l+1}{k+2}$.

Let the set of level $k$ characters $R_{k}(G) \subset R(G)$ be the additive subgroup generated by all $\chi_{\mu}$ with $\mu \in \Lambda_{k}^{*}$. One has the orthogonality relations,

$$
\begin{aligned}
\sum_{\lambda \in \Lambda_{k}^{*}}\left|J\left(t_{\lambda}\right)\right|^{2} \chi_{\mu}\left(t_{\lambda}\right) \chi_{\mu^{\prime}}\left(t_{\lambda}\right)^{*} & =\# T_{k+c} \delta_{\mu, \mu^{\prime}}, \quad \mu, \mu^{\prime} \in \Lambda_{k}^{*} \\
\sum_{\mu \in \Lambda_{k}^{*}}\left|J\left(t_{\lambda}\right)\right|^{2} \chi_{\mu}\left(t_{\lambda}\right) \chi_{\mu}\left(t_{\lambda^{\prime}}\right)^{*} & =\# T_{k+c} \delta_{\lambda, \lambda^{\prime}}, \quad \lambda, \lambda^{\prime} \in \Lambda_{k}^{*}
\end{aligned}
$$


where $J: T \rightarrow \mathbb{C}$ is the Weyl denominator

$$
J(t)=\sum_{w \in W}(-1)^{l(w)} t^{w \rho} .
$$

These formulas are obtained from the Weyl character formula and finite Fourier transform for $T_{k+c} \subset T$. As a consequence, every level $k$ character is determined by its restriction to $T_{k+c}^{\mathrm{reg}}$. One may view $R_{k}(G)$ as a quotient of $R(G)$ by the ideal of characters vanishing on $T_{k+c}^{\text {reg }}$; this defines a ring structure on $R_{k}(G)$ known as fusion product.

Let us return to the problem of calculating multiplicities in $\chi(M)$ from the fixed point formula. The remark following Theorem 2.1 shows that $\chi(M) \in R_{k}(G)$ provided $k_{j}>0$ are chosen large enough so that $\mathfrak{A}_{k}^{*}$ contains the moment polytope $\Phi(M) \cap \mathfrak{t}_{+}^{*}$. The multiplicity of any $\mu \in \Lambda_{k}^{*}$ can be computed from the orthogonality relation (5), substituting the Atiyah-SegalSinger fixed point formula for $\chi\left(M, t_{\lambda}\right)$. On the other hand this multiplicity equals $\chi\left(M_{\mu}\right)$, by Theorem 2.1. This gives,

Proposition 2.3. Let $(M, \omega, \Phi)$ be a pre-quantized compact Hamiltonian $G$-manifold with character $\chi(M)$ at level $k$. Then the index of the reduced space $M_{\mu}$ can be expressed in terms of fixed point contributions in $M$ :

$$
\chi\left(M_{\mu}\right)=\frac{1}{\# T_{k+c}} \sum_{\lambda \in \Lambda_{k}^{*}} \chi_{\mu}\left(t_{\lambda}\right)\left|J\left(t_{\lambda}\right)\right|^{2} \sum_{F \subseteq M^{t} \lambda} \chi\left(\nu_{F}, t_{\lambda}\right)^{*} .
$$

\section{Review of loop group actions and group-valued moment maps.}

\subsection{Loop groups.}

For the material in this subsection we refer to Pressley-Segal [31, Section 4.3]. Let $S^{1}=\mathbb{R} / \mathbb{Z}$ be the parametrized circle with coordinate $s$.

Throughout we will fix a "Sobolev level" $f>1$. We denote by $\underline{\Omega}^{0}\left(S^{1}, \mathfrak{g}\right)$ the space of $\mathfrak{g}$-valued 0 -forms of Sobolev class $f+1 / 2$ and by $\Omega^{1}\left(S^{1}, \mathfrak{g}\right)$ the $\mathfrak{g}$-valued 1-forms of Sobolev class $f-1 / 2$. Then forms in $\underline{\Omega}^{0}\left(S^{1}, \mathfrak{g}\right)$ are $C^{1}$ and those in $\Omega^{1}\left(S^{1}, \mathfrak{g}\right)$ are $C^{0}$. Let the (free) loop group $L G$ consist of maps $S^{1} \rightarrow G$ of Sobolev class $f+1 / 2$. It is a Banach Lie group with Lie algebra $L \mathfrak{g}=\underline{\Omega}^{0}\left(S^{1}, \mathfrak{g}\right)$. The kernel of the evaluation mapping $L G \rightarrow G, g \mapsto g(0)$ is called the based loop group $\Omega G$. The free loop group is a semi-direct product $L G=G \ltimes \Omega G$ where $G$ is embedded as constant loops and the action of $G$ 
on $\Omega G$ is pointwise conjugation. We embed the lattice $\Lambda \subset \mathfrak{t}$ into $L G$ by the map which takes $\xi \in \Lambda$ to the loop,

$$
\mathbb{R} / \mathbb{Z} \rightarrow G, \quad s \mapsto \exp (-s \xi) .
$$

The loop group $L G$ acts on the affine space $\mathcal{A}\left(S^{1}\right)=\underline{\Omega}^{1}\left(S^{1}, \mathfrak{g}\right)$ of connections on the trivial $G$-bundle over $S^{1}$ by gauge transformations

$$
g \cdot \mu=\operatorname{Ad}_{g}(\mu)-g^{*} \theta^{R} .
$$

Here $\theta^{R} \in \Omega^{1}(G, \mathfrak{g})$ denotes the right-invariant Maurer-Cartan form. The orbit space for this action can be described as follows. Consider the embedding $\mathfrak{t} \subset \mathfrak{g} \hookrightarrow \underline{\Omega}^{1}\left(S^{1}, \mathfrak{g}\right)$ by the map $\xi \mapsto \xi \mathrm{d} s$. The intersection of any $L G$-orbit with $\mathfrak{t}$ is an orbit of the affine Weyl group $W_{\text {aff }}=W \ltimes \Lambda$. Hence there are natural identifications,

$$
\underline{\Omega}^{1}\left(S^{1}, \mathfrak{g}\right) / L G=\mathfrak{t} / W_{\text {aff }}=T / W=G / \operatorname{Ad}(G) .
$$

In particular, one has a 1-1 correspondence between $L G$-orbits and conjugacy classes in $G$. For $G$ simply connected, all of these sets are also identified with the fundamental alcove $\mathfrak{A}$. That is, each coadjoint $L G$-orbit meets the alcove $\mathfrak{A}$ in exactly one point.

For $s \in \mathbb{R}$ and any $\mu \in \underline{\Omega}^{1}\left(S^{1}, \mathfrak{g}\right)$ let $\operatorname{Hol}_{s}(\mu) \in G$ denote the parallel transport from 0 to $s$. Thus $s \mapsto h(s)=\operatorname{Hol}_{s}(\mu)$ is the unique solution of the initial value problem $h^{\prime}(s) h(s)^{-1} \equiv h^{*} \theta^{R}=\mu, h(0)=e$. One has the equivariance property

$$
\operatorname{Hol}_{s}(g \cdot \mu)=g(s) \operatorname{Hol}_{s}(\mu) g(0)^{-1},
$$

showing in particular that the based gauge group $\Omega G$ acts freely. Let $\operatorname{Hol}(\mu):=\operatorname{Hol}_{1}(\mu)$ denote holonomy of $\mu$ around $S^{1}$. Any two elements $\mu_{1}, \mu_{2} \in \underline{\Omega}^{1}\left(S^{1}, \mathfrak{g}\right)$ with $\operatorname{Hol}\left(\mu_{1}\right)=\operatorname{Hol}\left(\mu_{2}\right)$ are related by a based gauge transformation, $\operatorname{Hol}_{s}\left(\mu_{1}\right) \operatorname{Hol}_{s}\left(\mu_{2}\right)^{-1}$. The holonomy map

$$
\text { Hol : } \underline{\Omega}^{1}\left(S^{1}, \mathfrak{g}\right) \rightarrow G
$$

gives $\underline{\Omega}^{1}\left(S^{1}, \mathfrak{g}\right)$ the structure of a Banach principal $\Omega G$-bundle over $G$. It is equivariant with respect to the evaluation map $L G \rightarrow G$ and once again gives the correspondence between $L G$-orbits and conjugacy classes.

For any $\mu \in \underline{\Omega}^{1}\left(S^{1}, \mathfrak{g}\right)$, the evaluation map $L G \rightarrow G$ induces an isomorphism, $L G_{\mu} \cong G_{\operatorname{Hol}(\mu)}$, with inverse map

$$
G_{\mathrm{Hol} \mu} \rightarrow L G_{\mu}, \quad g \mapsto \operatorname{Ad}_{\mathrm{Hol}_{s}(\mu)}(g) .
$$


In particular this shows that all stabilizer groups $L G_{\mu}$ are compact.

If we make the additional assumption that $G$ is simply connected, then all centralizers $G_{g}$, hence also all stabilizer groups $L G_{\mu} \cong G_{\mathrm{Hol}(\mu)}$, are connected. For any open face $\sigma \subset \mathfrak{A}$, the stabilizer group $L G_{\mu}$ of $\mu \in \sigma$ is independent of $\mu$ and will therefore be denoted $L G_{\sigma}$. The evaluation map defines an isomorphism $L G_{\sigma} \cong G_{\sigma}$ with the centralizer of $g=\exp (\mu)$. If $\sigma \subset \bar{\tau}$ then $L G_{\tau} \subset L G_{\sigma}$ and $G_{\tau} \subset G_{\sigma}$; in particular every $G_{\sigma}, L G_{\sigma}$ contains the maximal torus $T$. The root system $\mathfrak{R}_{\sigma}$ of $G_{\sigma}$ consists of all $\alpha \in \mathfrak{R}$ such that the restriction $\left.\alpha\right|_{\sigma}$ is integer valued. From the definition of the dual Coxeter number, it follows that $B_{c}^{\sharp}(\rho) \in \operatorname{int}(\mathfrak{A})$. Let $\mathfrak{R}_{+, \sigma}$ be all $\alpha \in \mathfrak{R}_{\sigma}$ such that $\alpha\left(B_{c}^{\sharp}(\rho)\right) \geq\left.\alpha\right|_{\sigma}$, and let $2 \rho_{\sigma}$ be their sum. One can check (cf. [28]) that

$$
\gamma_{\sigma}:=B_{c}^{\sharp}\left(\rho-\rho_{\sigma}\right)
$$

is always contained in $\sigma$. The positive Weyl chamber $\mathfrak{t}_{+, \sigma}$ for $\mathfrak{R}_{+, \sigma}$ is the cone over $\mathfrak{A}-\gamma_{\sigma}$.

\subsection{Hamiltonian loop group actions.}

The space $L \mathfrak{g}^{*}:=\Omega^{1}\left(S^{1}, \mathfrak{g}^{*}\right)$ is a dense sub-space of the topological dual space of $L \mathfrak{g}=\underline{\Omega}^{0}\left(S^{1}, \mathfrak{g}\right)$, using the pairing of $\mathfrak{g}^{*}$ and $\mathfrak{g}$ followed by integration over $S^{1}$. Given an invariant inner product $B$ on $\mathfrak{g}$, the isomorphism $B^{b}$ : $\mathfrak{g} \rightarrow \mathfrak{g}^{*}$ gives rise to an identification $B^{b}: \underline{\Omega}^{1}\left(S^{1}, \mathfrak{g}\right) \rightarrow L \mathfrak{g}^{*}$. The affine $L G$-action induced on $L \mathfrak{g}^{*}$ via this isomorphism will be called the coadjoint loop group action, and its orbits will be called coadjoint $L G$-orbits. Recall that a 2 -form on a Banach manifold $\widehat{M}$ is called weakly symplectic if its kernel is trivial everywhere.

Definition 3.1. A Hamiltonian $L G$-manifold is a triple $(\widehat{M}, \widehat{\omega}, \widehat{\Phi})$, consisting of a Banach manifold $\widehat{M}$ with a smooth $L G$-action, a weakly symplectic 2 -form $\widehat{\omega}$ on $\widehat{M}$, and an equivariant map $\widehat{\Phi}: \widehat{M} \rightarrow L \mathfrak{g}^{*}$ satisfying

$$
\iota\left(\xi_{\widehat{M}}\right) \widehat{\omega}=\mathrm{d} \widehat{\Phi}(\xi), \quad \xi \in L \mathfrak{g} .
$$

Much of the theory of compact Hamiltonian $G$-spaces carries over to Hamiltonian loop group spaces if one assumes that the moment map $\widehat{\Phi}$ is proper. For example, if $\mu \in L \mathfrak{g}^{*}$ is a regular value of the moment map then the reduced space $\widehat{M}_{\mu}=\widehat{\Phi}^{-1}(\mu) / L G_{\mu}$ is a compact, finite dimensional symplectic orbifold. More generally, this holds true for quasi-regular values $\mu$, i.e. if all $L G_{\mu}$-orbits in $\widehat{\Phi}^{-1}(\mu)$ have the same dimension. 
For $G$ simply connected and $\widehat{M}$ connected, there is also a convexity theorem [30, Theorem 4.11], stating the intersection $\widehat{\Phi}(\widehat{M}) \cap \mathfrak{A}$ is a convex polytope. We refer to this polytope as the moment polytope of $\widehat{M}$.

Basic examples for Hamiltonian $L G$-spaces with proper moment maps are coadjoint orbits $\mathcal{O}=L G \cdot \mu$ for $\mu \in L \mathfrak{g}^{*}$, with moment map the inclusion. The 2-form is uniquely determined by the moment map condition, and is given by an analog to the Kirillov-Kostant-Souriau formula. The motivating examples of moduli spaces of flat bundleson surfaces with boundary are discussed in Section 5.

\subsection{Group-valued moment maps.}

Suppose $(\widehat{M}, \widehat{\omega}, \widehat{\Phi})$ is a Hamiltonian $L G$-space with proper moment map. As mentioned above, the holonomy map Hol $: L \mathfrak{g}^{*} \rightarrow G$ is a principal $\Omega G$-bundle. By equivariance of the moment map, the based loop group $\Omega G$ also acts freely on $\widehat{M}$, and by properness the quotient $M:=\widehat{M} / \Omega G$ is a compact, smooth, finite dimensional manifold [29, Section 3.2.1]. The action of $L G=G \ltimes \Omega G$ descends to a $G$-action on $M$, and the moment map $\widehat{\Phi}$ to a $G$-equivariant map $\Phi: M \rightarrow G$, which makes the following diagram commute:

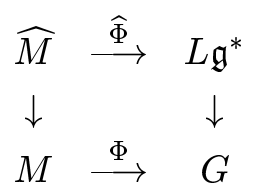

We call $M$ the holonomy manifold of $\widehat{M}$. In [1] $M$ is interpreted as a Hamiltonian $G$-space with group valued moment map $\Phi$. The definition is as follows. Let $\theta^{L}, \theta^{R}$ be the left resp. right invariant Maurer-Cartan forms on $G$, and $\eta \in \Omega^{3}(G)$ the canonical closed, bi-invariant 3-form

$$
\eta=\frac{1}{12} B\left(\theta^{L},\left[\theta^{L}, \theta^{L}\right]\right)=\frac{1}{12} B\left(\theta^{R},\left[\theta^{R}, \theta^{R}\right]\right) .
$$

Define a 2 -form on $L \mathfrak{g}^{*}$ by

$$
\varpi=\frac{1}{2} \int_{0}^{1} B\left(\operatorname{Hol}_{s}^{*} \theta^{R}, \frac{\partial}{\partial s} \operatorname{Hol}_{s}^{*} \theta^{R}\right) \mathrm{d} s .
$$

By [1, Proposition 8.1] the form $\varpi$ has the property $\mathrm{d} \varpi=\mathrm{Hol}^{*} \eta$, and its contractions with generating vector fields for the $L G$-action are

$$
\iota\left(\xi_{L \mathfrak{g}^{*}}\right) \varpi=-\mathrm{d} \mu(\xi)+\frac{1}{2} \mathrm{Hol}^{*} B\left(\theta^{L}+\theta^{R}, \xi(0)\right) .
$$


Here $\mu(\xi)$ is the function on $L \mathfrak{g}^{*}$ taking $\mu \in L \mathfrak{g}^{*}$ to the pairing with $\xi \in L \mathfrak{g}$. It follows that $\widehat{\omega}-\widehat{\Phi}^{*} \varpi \in \Omega^{2}(\widehat{M})=\operatorname{Hol}^{*} \omega$ for a unique 2-form $\omega \in \Omega^{2}(M)$. As shown in [1], this 2-form has properties

$$
\begin{gathered}
\mathrm{d} \omega=\Phi^{*} \eta, \\
\iota\left(\xi_{M}\right) \omega=\frac{1}{2} \Phi^{*} B\left(\theta^{L}+\theta^{R}, \xi\right), \quad \text { for all } \xi \in \mathfrak{g}, \\
\operatorname{ker}\left(\omega_{m}\right)=\left\{\xi_{M}(m) \mid \operatorname{Ad}_{\Phi(m)} \xi=-\xi\right\}
\end{gathered}
$$

and conversely every compact $G$-manifold $M$ with an equivariant map $\Phi \in$ $C^{\infty}(M, G)$ and an invariant 2 -form $\omega$ with these three properties defines a Hamiltonian $L G$-manifold with proper moment map. We call $(M, \omega, \Phi)$ with properties (12), (13), (14) a group-valued Hamiltonian $G$-space. As for $\mathfrak{g}^{*}$-valued moment maps, we call an element $g \in G$ a quasi-regular value of $\Phi$ if all $G_{g^{-}}$orbits in $\Phi^{-1}(g)$ have the same dimension. It is proved in [1] that in this case the reduced space $M_{g}=\Phi^{-1}(g) / G_{g}$ is a symplectic orbifold, with 2 -form induced from $\omega$. Given $\mu \in L \mathfrak{g}^{*}$ with $g=\operatorname{Hol}(\mu)$, one finds that $g$ is quasi-regular for $\Phi$ if and only if $\mu$ is quasi-regular for $\widehat{\Phi}$, and $\widehat{M}_{\mu}=M_{g}$ as symplectic spaces.

\section{The fixed point formula for loop group actions.}

In this Section, we discuss pre-quantization of Hamiltonian loop group manifolds and state the main result of this paper, the fixed point formula Theorem 4.3 .

\subsection{Central extension of $L G$.}

We will assume for the rest of this paper that $G$ is simply connected, and let $G=G_{1} \times \ldots \times G_{s}$ be its decomposition into simple factors. Each of the bilinear forms $B=B_{l}, l \in \mathbb{R}^{s}$, on $\mathfrak{g}$ defines a central extension $\widehat{\mathrm{Lg}}$ of $L \mathfrak{g}=\underline{\Omega}^{0}\left(S^{1}, \mathfrak{g}\right)$ by $\mathbb{R}$, with cocycle

$$
\mathfrak{c}: L \mathfrak{g} \times L \mathfrak{g} \rightarrow \mathbb{R}, \mathfrak{c}\left(\xi_{1}, \xi_{2}\right)=\int_{S^{1}} B\left(\xi_{1}, \mathrm{~d} \xi_{2}\right) .
$$

The dual action of $L G$ on $\widehat{L \mathfrak{g}}^{*}=\underline{\Omega}^{1}\left(S^{1}, \mathfrak{g}^{*}\right) \times \mathbb{R}$ is given by the formula, $g \cdot(\mu, \tau)=\left(\mu-\tau B^{b}\left(g^{*} \theta^{R}\right), \tau\right)$. If $B$ is non-degenerate, it identifies $\widehat{L \mathfrak{g}}^{*}=$ 
$\underline{\Omega}^{1}\left(S^{1}, \mathfrak{g}\right) \times \mathbb{R}$ and the gauge action of $L G$ on $\mathcal{A}\left(S^{1}\right)$ becomes the action on the affine hyperplane $\tau=1$.

It is known [31, Theorem (4.4.1)] that the Lie algebra extension exponentiates to a group extension

$$
1 \rightarrow \mathrm{U}(1) \rightarrow \widehat{L G} \rightarrow L G \rightarrow 1
$$

exactly if all $l_{j}$ are integers. Since $L G$ is connected and simply connected, the extension is unique. For any subgroup $\mathcal{H} \subset L G$, we denote by $\widehat{\mathcal{H}}$ the pull-back of the central extension. Since the defining cocycle $\mathfrak{c}$ vanishes on $\mathfrak{g}$, and since $G$ is connected and simply connected, the central extension $\widehat{G}$ is canonically trivial. That is, there is an embedding

$$
G \rightarrow \widehat{L G}
$$

and $\widehat{L G}$ is a semi-direct product $\widehat{L G}=G \ltimes \widehat{\Omega G}$. The following proposition describes the central extensions for various subgroups of $L G$.

Proposition 4.1. Let $l \in \mathbb{Z}^{s}$ and $\widehat{L G}=\widehat{L G}^{(l)}$ the corresponding central extension of the loop group.

(a) Let $\xi, \xi^{\prime} \in \Lambda$, and $\hat{\xi}, \hat{\xi}^{\prime} \in \widehat{\Lambda}$ arbitrary lifts. Then the Lie group commutator is given by the formula

$$
\left[\hat{\xi}, \hat{\xi}^{\prime}\right]=(-1)^{B_{l}\left(\xi, \xi^{\prime}\right)} .
$$

In particular, if all $l_{j}$ are even, the central extension $\widehat{\Lambda}$ is trivial.

(b) Embed $T \hookrightarrow \widehat{L G}$ using (16). The central extension of $L T \subset L G$ is a semi-direct product

$$
\widehat{L T}=T \ltimes \widehat{\Omega T}
$$

where the action of $T$ on $\widehat{\Omega T}$, over the connected component of $\Omega T$ containing $\xi \in \Lambda$, is given by multiplication with $t^{B^{b}}(\xi)$.

(c) Suppose all $l_{j}>0$. The central extension of $T_{l} \times \Omega T$ is a direct product,

$$
\widehat{T_{l} \times \Omega T}=T_{l} \times \widehat{\Omega T} .
$$

Proof. Part (a) is proved by Pressley-Segal [31, Section 4.8] for simply laced groups, and by Toledano Laredo [35, Proposition 3.1] in the general case. Part (c) follows from part (b) since $t^{B^{b}(\xi)}=1$ for $\xi \in \Lambda, t \in T_{l}$. 
It remains to prove part (b). Since the conjugation action of $T$ on $\Omega T$ is trivial, its action on any connected component of $\widehat{\Omega T}$ is scalar multiplication on the fibers by some character for $T$. To compute the weight for this action at $\xi \in \Lambda \subset \Omega T$, let $\alpha \in \Omega^{1}(\widehat{L G})$ be the left-invariant connection 1-form defined by the splitting $\widehat{L \mathfrak{g}}=L \mathfrak{g} \times \mathbb{R}$. The weight $\mu \in \Lambda^{*}$ for the $T$-action on the fiber over $\xi$ is given by

$$
\langle\mu, \zeta\rangle=\left\langle\alpha_{\hat{\xi}}, \zeta_{\widehat{L G}}\right\rangle, \quad \zeta \in \mathfrak{t}
$$

where $\hat{\xi}$ is any lift of $\xi$. In left trivialization of the cotangent bundle of $\widehat{L G}, \alpha$ is the constant map from $\widehat{L G}$ to $(0,1) \in \widehat{L \mathfrak{g}}^{*}$. This shows that its contraction with the left-invariant vector field generated by $\zeta$ is zero. To compute its contractions with the right-invariant vector field generated by $\zeta$, we note that under the right action of $\hat{\xi}$,

$$
R_{\hat{\xi}^{-1}}^{*} \alpha=\operatorname{Ad}_{\hat{\xi}}^{*} \alpha=\hat{\xi} \cdot(0,1)=\left(B^{b}(\xi), 1\right)
$$

Since $\zeta_{\widehat{L G}}$ is the difference between left and right invariant vector fields, we find that $\left\langle\alpha_{\hat{\xi}}, \zeta_{\overparen{L G}}\right\rangle=\left\langle B^{b}(\xi), \zeta\right\rangle$, proving $\mu=B^{b}(\xi)$.

Below we will often use the following terminology. A level l line bundle over an $L G$-space $X$ is an $\widehat{L G}^{(l)}$-equivariant line bundle $L \rightarrow X$ where the central $\mathrm{U}(1)$ acts by scalar multiplication with weight 1 . Equivalently, $L$ carries actions of the central extensions $\widehat{L G}_{j}^{(1)}$ where the central U(1)'s act with weights $l_{j}$. The tensor product of two line bundles at levels $l, l^{\prime}$ is at level $l+l^{\prime}$, and line bundles at level 0 are simply $L G$-equivariant line bundles.

\subsection{Fixed point formula for Hamiltonian loop group actions.}

Suppose now that $B=B_{k}$ where all $k_{j}$ are positive integers, and let $\widehat{L G}=\widehat{L G}^{(k)}$. Using $B$ we identify $\mathfrak{g} \cong \mathfrak{g}^{*}$ and $\underline{\Omega}^{1}\left(S^{1}, \mathfrak{g}\right) \cong L \mathfrak{g}^{*}$. The formula for the coadjoint action of $L G$ on $\widehat{L \mathfrak{g}}^{*}$ shows that a Hamiltonian $L G$-manifold $(\widehat{M}, \widehat{\omega}, \widehat{\Phi})$ is equivalently a Hamiltonian $\widehat{L G}$-manifold on which the central $\mathrm{U}(1)$ acts trivially, with moment map 1. Accordingly we define a pre-quantum line bundle to be a level $k$ line bundle $L \rightarrow \widehat{M}$ with invariant connection, such that the Chern form $c_{1}(L)$ is equal to the symplectic form $\widehat{\omega}$ and such that the vertical part of the fundamental vector fields is given by Kostant's formula. 
Example 4.2. The coadjoint orbit $L G \cdot \mu$ through $\mu \in \mathfrak{A}_{k}^{*}$ is pre-quantizable if and only if $\mu \in \Lambda_{k}^{*}$. The pre-quantum line bundle is the associated bundle $\widehat{L G} \times \widehat{L G}_{\mu} \mathbb{C}_{(\mu, 1)}$. If $\Sigma$ is a compact oriented surface with boundary, the moduli space $\mathcal{M}(\Sigma)$ of flat $G$-connections by based gauge equivalence is pre-quantizable. (For non-simply connected groups the situation is more complicated.)

Suppose the moment map $\widehat{\Phi}$ is proper and $\mu \in \Lambda_{k}^{*}$ is a quasi-regular value. Then $\widehat{M}_{\mu}$ is a symplectic orbifold with pre-quantum orbifold line bundle

$$
L_{\mu}=\left(\left.L\right|_{\widehat{\Phi}^{-1}(\mu)} \otimes \mathbb{C}_{-(\mu, 1)}\right) / \widehat{L G}_{\mu} .
$$

(Notice that in fact $L G_{\mu}$ acts on the tensor product, for the central $\mathrm{U}(1) \subset \widehat{L G}_{\mu}$ acts with weights +1 and -1 on the two factors and hence acts trivially on the product.) Hence the $\operatorname{Spin}_{c}$-index $\chi\left(\widehat{M}_{\mu}\right)$ is defined for quasi-regular $\mu \in \Lambda_{k}^{*}$. If $\mu$ is not quasi-regular one can still define $\chi\left(\widehat{M}_{\mu}\right)$ by

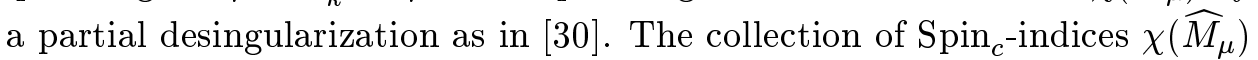
is determined by the restriction of the level $k$ character $\sum_{\mu \in \Lambda_{k}^{*}} \chi\left(\widehat{M}_{\mu}\right) \chi_{\mu}$ to $T_{k+c}^{\mathrm{reg}}$.

The main result of the paper is the following loop group analog of Equation (7).

Theorem 4.3 (Fixed Point Formula). Suppose $(\widehat{M}, \widehat{\omega}, \widehat{\Phi})$ is a pre-quantized Hamiltonian LG-manifold with proper moment map. For all $t \in$ $T_{k+c}^{\mathrm{reg}}$,

$$
\sum_{\mu \in \Lambda_{k}^{*}} \chi\left(\widehat{M}_{\mu}\right) \chi_{\mu}(t)=\sum_{F \subseteq M^{t}} \chi\left(\nu_{F}, t\right)
$$

Here the right hand side is a sum over components of the fixed point set for the action of $t$ on $M=\widehat{M} / \Omega G$, and the fixed point contributions have the form

$$
\chi\left(\nu_{F}, t\right)=\zeta_{F}(t)^{1 / 2} \int_{F} \frac{\widehat{A}(F) e^{\frac{1}{2} c_{1}\left(\mathcal{L}_{F}\right)}}{\mathcal{D}_{\mathbb{R}}\left(\nu_{F}, t\right)} .
$$

The terms entering the integral (18) will be explained in Subsection 4.3 below.

Using the orthogonality relations for level $k$ characters, the Theorem shows that for all $\mu \in \Lambda_{k}^{*}$,

$$
\chi\left(\widehat{M}_{\mu}\right)=\frac{1}{\# T_{k+c}} \sum_{\lambda \in \Lambda_{k}^{*}} \chi_{\mu}\left(t_{\lambda}\right)\left|J\left(t_{\lambda}\right)\right|^{2} \sum_{F \subseteq M^{t_{\lambda}}} \chi\left(\nu_{F}, t_{\lambda}\right)^{*} .
$$


The proof of Theorem 4.3 will be given in the final Section of this paper.

\subsection{The fixed point contributions.}

In general, the holonomy manifold $M$ does not carry a naturally induced Spin $_{c}$-structure, even though the expressions (18) resemble the fixed point contributions of a $\operatorname{Spin}_{c}$-Dirac operator. Our strategy for defining the terms entering (18) is to first restrict data on $\widehat{M}$ to a certain finite dimensional submanifold $\widetilde{F} \subset \widehat{M}^{t}$ covering $F$, and then to show that the restrictions descend to $F$ itself.

Proposition 4.4. Let $(M, \omega, \Phi)$ be a Hamiltonian $G$-space with group valued moment map, and $(\widehat{M}, \widehat{\omega}, \widehat{\Phi})$ the corresponding loop group space. Let $t \in T^{\mathrm{reg}}$, and $F \subseteq M^{t}$ a connected component of the fixed point set. Let $\widehat{F}$ be the pre-image of $F$ under the map $\widehat{M}^{t} \rightarrow M^{t}$, and $\widetilde{F}$ the intersection of $\widehat{F}$ with $\widehat{\Phi}^{-1}(\mathfrak{t})$.

(a) $F$ is a group-valued Hamiltonian $T$-space, with symplectic form $\omega_{F}$ and moment map $\Phi_{F}$ the pull-backs of $\omega, \Phi$.

(b) $\widehat{F}$ is a (possibly disconnected) Hamiltonian LT-manifold, with 2-form and moment map the pull-backs of $\widehat{\omega}, \widehat{\Phi}$. It has $\left(F, \omega_{F}, \Phi_{F}\right)$ as its holonomy manifold.

(c) $\widetilde{F}$ is a finite-dimensional Hamiltonian T-manifold, with 2-form and moment map the pull-backs of $\widehat{\omega}, \widehat{\Phi}$. It carries a free symplectic action of the lattice $\Lambda$ which commutes with the action of $T$. One has $F=$ $\widetilde{F} / \Lambda$ as a symplectic $T$-manifold, and $\widetilde{F}=\widehat{F} / \Omega_{0} T$ where $\Omega_{0} T$ is the identity component of $\Omega T$.

Proof. We begin by showing that $F$ is symplectic. Since $t$ is a regular element, $\Phi(F) \subseteq G^{t}=T$. Let $m \in F, g=\Phi(m)$, and consider the splitting of the tangent space

$$
T_{m} M=E \oplus \mathfrak{g}_{g}^{\perp}
$$

where $E=\left(\mathrm{d}_{m} \Phi\right)^{-1}\left(\mathfrak{g}_{g}\right)$ and the second summand is embedded by the generating vector fields. By $[1$, Section 7$]$, the splitting is $\omega$-orthogonal and the restriction of $\omega$ to $E$ is symplectic. Since the action of $t$ on $E$ preserves the 2-form, the subspace $T_{m} F=\left(T_{m} M\right)^{t}=E^{t}$ is symplectic as well. This shows that $\omega_{F}$ is non-degenerate. It is closed since $\mathrm{d} \omega_{F}=$ 
$\iota_{F}^{*} \Phi^{*} \eta=\Phi_{F}^{*} \iota_{T}^{*} \eta=0$. The moment map condition for $\left(F, \omega_{F}, \Phi_{F}\right)$ follows from that for $(M, \omega, \Phi)$. This proves (a).

Clearly $\widehat{F}$ is $\Omega T$-invariant, and its image under the moment map is contained in $\left(L \mathfrak{g}^{*}\right)^{t}=L \mathfrak{t}^{*}$. Also $\widehat{F} / \Omega T \subseteq F$. To prove the reverse inclusion, suppose $m \in F$. Its pre-image under the map $\widehat{M} \rightarrow M$ meets $\widehat{\Phi}^{-1}\left(L \mathrm{t}^{*}\right)$. We need to show that any pre-image $\hat{m} \in \widehat{\Phi}^{-1}\left(L t^{*}\right)$ is fixed under $t$. By definition of the action on $M$, any pre-image satisfies $t \cdot \hat{m}=g \cdot \hat{m}$ for some $g \in \Omega G$. By equivariance and since $T$ acts trivially on $L \mathfrak{t}^{*}$, this means $\widehat{\Phi}(\hat{m})=g \cdot \widehat{\Phi}(\hat{m})$. Since $\Omega G$ acts freely, we conclude $g=e$ and therefore $t \cdot \hat{m}=\hat{m}$. Viewing $L t^{*} \rightarrow T$ as a principal $\Omega T$-bundle, $\widehat{F} \rightarrow F$ is the pull-back bundle under the map $\Phi_{F}: F \rightarrow T$. From the constructions, it follows that $\widehat{F}$ is the Hamiltonian $L T$-manifold associated to $F$, proving (b).

Now view $\mathfrak{t} \rightarrow T$ as a $\Lambda$-principal sub-bundle of $L \mathfrak{t}^{*} \rightarrow T$. We have $L \mathfrak{t}^{*} / \Omega_{0} T=\mathfrak{t}$ since every $\Omega_{0} T$-orbit in $L \mathfrak{t}^{*}$ passes through a unique point in t. Then (c) follows since $\widehat{F} \rightarrow F$ and $\widetilde{F} \rightarrow F$ are pull-back bundles with respect to $\Phi_{F}: F \rightarrow T$, and from the fact that the form $\varpi \in \Omega^{2}\left(L \mathfrak{g}^{*}\right)$ vanishes if pulled back to $\mathfrak{t} \subset L \mathfrak{t}^{*}$.

Remark 4.5. By a similar argument, the fixed point set $M^{g}$ of any group element $g \in G$ is a group valued Hamiltonian $G_{g}$-manifold, with the pullbacks of $\omega$ and $\Phi$ as 2-form and moment map.

Under the assumptions of Theorem 4.3, we are now going to explain the ingredients of the fixed point contributions (18). First, we will define a level $2(k+c)$ "Spin c" line bundle $\mathcal{L}:=L^{2} \otimes K^{-1} \rightarrow \widehat{M}$, where $K^{-1}$ is the "anticanonical line bundle" $K^{-1}$ for Hamiltonian loop group manifolds [28]. As we will explain, the restriction $\left.\mathcal{L}\right|_{\widetilde{F}}$ descends to a $T_{2(k+c)^{-}}$equivariant line bundle $\mathcal{L}_{F} \rightarrow F$. The element $t$ acts on $\mathcal{L}_{F}$ as scalar multiplication by some element $\zeta_{F}(t)$, and we will show how to choose a square root.

To carry out the details, we need the symplectic cross-section theorem for Hamiltonian $L G$-manifolds, cf. [30]. It is an analog of the GuilleminSternberg cross-section theorem [20] for compact groups. For any vertex $\sigma$ of $\mathfrak{A}$, let $\mathfrak{A}_{\sigma} \subset \mathfrak{A}$ denote the complement of the closed face opposite $\sigma$. Given an arbitrary open face $\sigma$, define $\mathfrak{A}_{\sigma}$ to be the intersection of all $\mathfrak{A}_{\tau}$ with $\tau$ a vertex of $\bar{\sigma}$. Then the flow-outs

$$
U_{\sigma}:=L G_{\sigma} \cdot \mathfrak{A}_{\sigma} \subset \underline{\Omega}^{1}\left(S^{1}, \mathfrak{g}\right) \cong L \mathfrak{g}^{*}
$$

are smooth, finite dimensional submanifolds, and are slices for points in $\sigma$. The maps $L G \times{ }_{L G_{\sigma}} U_{\sigma} \rightarrow L \mathfrak{g}^{*}$ are embeddings as open subsets and their images form an open cover. The cross-section theorem states that the 
pre-images $Y_{\sigma}=\widehat{\Phi}^{-1}\left(U_{\sigma}\right)$ are $L G_{\sigma^{-}}$invariant, finite dimensional symplectic submanifolds, and are Hamiltonian $\widehat{L G}_{\sigma}$-manifolds with the restriction $\widehat{\Phi}_{\sigma}$ of $\widehat{\Phi}$ as a moment map. (The central circle $\mathrm{U}(1) \subset \widehat{L G}_{\sigma}$ acts trivially, with moment map 1.) If $L \rightarrow \widehat{M}$ is a pre-quantum line bundle, it restricts

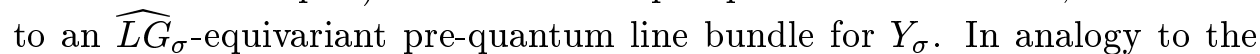
finite dimensional setting, we define a "Spin ${ }_{c}$ "-line bundle $\mathcal{L} \rightarrow \widehat{M}$ as a tensor-product of $L^{2}$ with an anti-canonical line bundle $K^{-1} \rightarrow \widehat{M}$. The notion of anti-canonical line bundle for Hamiltonian loop group manifolds was introduced in [28]. $K^{-1}$ is a level $2 c$ line bundle with the property that for each cross-section $Y_{\sigma}$, there is an $\widehat{L G}_{\sigma}^{(2 c)}$-equivariant isomorphism

$$
\left.K^{-1}\right|_{Y_{\sigma}} \cong K_{\sigma}^{-1} \otimes \mathbb{C}_{2\left(\rho-\rho_{\sigma}, c\right)}
$$

where $K_{\sigma}$ is the canonical line bundle for $Y_{\sigma}$. Here we are using that since $\gamma_{\sigma}=B_{c}^{\sharp}\left(\rho-\rho_{\sigma}\right)$ is contained in $\sigma$, the weight $2\left(\rho-\rho_{\sigma}, c\right)$ defines a 1 dimensional representation of $\widehat{L G}_{\sigma}^{(2 c)}$. The conditions (19) are consistent because for $\sigma \subset \bar{\tau}$, there is an $L G_{\tau^{-}}$equivariant isomorphism

$$
\left.K_{\sigma}^{-1}\right|_{Y_{\tau}}=K_{\tau}^{-1} \otimes \mathbb{C}_{2\left(\rho_{\tau}-\rho_{\sigma}\right)}
$$

Assume $t \in T^{\mathrm{reg}}$ and let $F \subseteq M^{t}$ be a connected component of the fixed point set. By part (c) of Proposition 4.1, the action of $\widehat{L G}^{(2(k+c))}$ restricts to an action of $T_{2(k+c)} \times \widehat{\Lambda}^{(2(k+c))}$, and furthermore by part (a) the central extension $\widehat{\Lambda}^{(2(k+c))}$ is trivial. Choosing any trivialization (by choosing lifts generators of $\Lambda$ ), we obtain a $T_{2(k+c)}$-equivariant line bundle $\mathcal{L}_{F} \rightarrow F$, by setting $\mathcal{L}_{F}:=\left.\mathcal{L}\right|_{\widetilde{F}} / \Lambda$.

Suppose now that $t \in T_{k+c}^{\mathrm{reg}}$ and let $\zeta_{F}(t)$ be the eigenvalue for the action on $\mathcal{L}_{F}$. We show how to specify a square root $\zeta_{F}(t)^{1 / 2}$. Let

$$
\zeta_{\widetilde{F}}(t), \mu_{\widetilde{F}}(t), \kappa_{\widetilde{F}}(t): \widetilde{F} \rightarrow \mathrm{U}(1)
$$

be the (locally constant) eigenvalues for the action of $t$ on $\left.\mathcal{L}\right|_{\widetilde{F}},\left.L\right|_{\widetilde{F}},\left.K\right|_{\widetilde{F}}$, respectively. Then $\zeta_{\widetilde{F}}(t)=\mu_{\widetilde{F}}(t)^{2} \kappa_{\widetilde{F}}(t)^{-1}$. In order to define the square root of $\zeta_{\widetilde{F}}(t)$ we need to define the square root $\kappa_{\widetilde{F}}(t)^{-1 / 2}$. Given a face $\sigma$ of $\mathfrak{A}$ and $w \in W_{\text {aff }}$ let $Y_{w \sigma}:=g \cdot Y_{\sigma}$, where $g \in N_{G}(T) \ltimes \Lambda \subset L G$ represents $w$. It is a finite dimensional symplectic submanifold, invariant under the action of $L G_{w \sigma}:=\operatorname{Ad}_{g}\left(L G_{\sigma}\right)$. Then

$$
\widehat{\Phi}^{-1}(\mathfrak{t}) \subset \bigcup_{\sigma \subset \mathfrak{A}} \bigcup_{w \in W_{\mathrm{aff}}} Y_{w \sigma}
$$


so that the intersections $Y_{w \sigma} \cap \widetilde{F}$ cover $\widetilde{F}$. By (19), if $\kappa_{\widetilde{F}}^{w \sigma}(t)^{-1}$ is the eigenvalue for the action on the anti-canonical line bundle for $Y_{w \sigma}$,

$$
\left.\kappa_{\widetilde{F}}(t)^{-1}\right|_{Y_{w \sigma} \cap \widetilde{F}}=\kappa_{\widetilde{F}}^{w \sigma}(t)^{-1} t^{2 w\left(\rho-\rho_{\sigma}\right)}
$$

where $w\left(\rho-\rho_{\sigma}\right)$ is defined using the level $c$ action of $W_{\text {aff }}$. As in Section 2.3 we can define the square root of $\kappa_{\widetilde{F}}^{w \sigma}(t)^{-1}$.

Lemma 4.6. There exists a unique locally constant $\mathrm{U}(1)$-valued function $\kappa_{\widetilde{F}}(t)^{-1 / 2}$ on $\widetilde{F}$ such that

$$
\left.\kappa_{\widetilde{F}}(t)^{-1 / 2}\right|_{Y_{w \sigma} \cap \widetilde{F}}=(-1)^{\epsilon(w, \sigma)+l(w)} \kappa_{\widetilde{F}}^{w \sigma}(t)^{-1 / 2} e^{2 \pi i\left\langle w\left(\rho-\rho_{\sigma}\right), v\right\rangle} .
$$

Here $v \in \mathfrak{t}$ is the unique vector in $W \cdot \mathfrak{A}$ with $\exp (v)=t, l(w)$ is the length of $w$, and $\epsilon(w, \sigma)$ is the number of positive roots $\alpha \in \mathfrak{R}_{+, \sigma}$ of $G_{\sigma}$ (cf. Section 3.1) such that $\left\langle w_{1} \alpha, v\right\rangle<0$, where $w_{1} \in W$ is the image of $w$ under the quotient map $W_{\mathrm{aff}} \rightarrow W$. Under the action of $\xi \in \Lambda$,

$$
\xi^{*} \kappa_{\tilde{F}}(t)^{-1 / 2}=t^{B_{c}^{\mathrm{b}}(\xi)} \kappa_{\tilde{F}}(t)^{-1 / 2} .
$$

Proof. Note first of all that the right hand side of (20) is well-defined. Indeed, if $w$ is replaced by $w^{\prime}$ with $w \sigma=w^{\prime} \sigma$, the factor $e^{2 \pi i\left\langle w\left(\rho-\rho_{\sigma}\right), v\right\rangle}$ does not change because $\rho-\rho_{\sigma} \in B_{c}^{b}(\sigma)$ is fixed under the level $c$ action of any element of $W_{\text {aff }}$ fixing $\sigma$, and $l(w)+\epsilon(w, \sigma)$ changes by an even number. Given faces $\sigma \subset \bar{\tau}$ of the alcove and any $w \in W_{\text {aff }}$, the symplectic normal bundle of $Y_{w \tau}$ inside $Y_{w \sigma}$ is $T \subset L G$-equivariantly isomorphic to $\mathfrak{g}_{\sigma} / \mathfrak{g}_{\tau}$, with $T$ acting via the isomorphism $w_{1}^{-1}: T \rightarrow T$ induced by $w_{1}$. Using the sign convention from Section 2.3, the square root of the eigenvalue for the action of $t=\exp (v)$ on $\mathfrak{g}_{\sigma} / \mathfrak{t}$ is given by $(-1)^{\epsilon(w, \sigma)} e^{2 \pi i\left\langle\rho_{\sigma}, w_{1}^{-1} v\right\rangle}$, and similarly for the action on $\mathfrak{g}_{\tau} / \mathfrak{t}$. Therefore,

$$
(-1)^{\epsilon(w, \sigma)} \kappa_{\tilde{F}}^{w \sigma}(\tilde{m}, t)^{-1 / 2}=(-1)^{\epsilon(w, \tau)} \kappa_{\tilde{F}}^{w \tau}(\tilde{m}, t)^{-1 / 2} e^{2 \pi i\left\langle w_{1}\left(\rho_{\tau}\right)-w_{1}\left(\rho_{\sigma}\right), v\right\rangle} .
$$

Since $w_{1}\left(\rho_{\tau}\right)-w_{1}\left(\rho_{\sigma}\right)=-w\left(\rho-\rho_{\tau}\right)+w\left(\rho-\rho_{\sigma}\right)$, we have shown that the right hand sides of equation (20) patch together to a well-defined locally constant function on $\widetilde{F}$.

The action of $\xi \in \Lambda \subset W_{\text {aff }}$ amounts to replacing $w$ by $\xi \cdot w$. This does not change $\epsilon(w, \tau)$, and changes $l(w)$ by an even number. The factor $e^{2 \pi i\left\langle w\left(\rho-\rho_{\sigma}\right), v\right\rangle}$ changes by $t^{B_{c}^{\mathrm{b}}(\xi)}$. 
Using Lemma 4.6, we define

$$
\zeta_{\widetilde{F}}(t)^{1 / 2}:=\mu_{\widetilde{F}}(t) \kappa_{\widetilde{F}}(t)^{-1 / 2} .
$$

Under the action of $\xi \in \Lambda$ it transforms according to $\xi^{*} \zeta_{\widetilde{F}}(t)^{1 / 2}=$ $t^{B_{c+k}^{\mathrm{b}}(\xi)} \zeta_{\widetilde{F}}(t)^{1 / 2}$. But $t^{B_{c+k}^{\mathrm{b}}(\xi)}=1$ since $t \in T_{k+c}$. Hence $\zeta_{\widetilde{F}}(t)^{1 / 2}$ is actually a constant, which defines $\zeta_{F}(t)^{1 / 2}$.

Remark 4.7. The following special case of the definition will be used in our applications to Verlinde formulas. Suppose that $F$ contains a point $m \in$ $\Phi^{-1}(e)$, and let $\tilde{m} \in \widetilde{F}$ be the unique point in the zero level set mapping to $m$. Then the tangent space $T_{m} M$ is symplectic, and the quotient map $\widehat{M} \rightarrow M$ induces a $t$-equivariant isomorphism of symplectic vector spaces, $T_{\tilde{m}} Y_{\{0\}} \cong$ $T_{m} M$. Hence, choosing any $t$-invariant compatible complex structure on $T_{m} M$ and letting $A(t) \in \operatorname{Aut}_{\mathbb{C}}\left(T_{m} M\right)$ denote the action of $t$,

$$
\kappa_{\widetilde{F}}(t, \tilde{m})^{-1 / 2}=\operatorname{det}_{\mathbb{C}}\left(A(t)^{1 / 2}\right) .
$$

If we know in addition that $t$ acts trivially on the fiber $L_{\tilde{m}}$ (e.g., if $t$ is in the identity component of $L G_{\tilde{m}}$ ), we obtain

$$
\zeta_{F}(t)^{1 / 2}=\operatorname{det}_{\mathbb{C}}\left(A(t)^{1 / 2}\right)
$$

with no explicit reference to the loop group space.

\subsection{Alternative version of the fixed point expressions.}

The expression for the fixed point contribution of $t \in T_{k+c}^{\mathrm{reg}}$ simplifies if for some $\sigma \subset \mathfrak{A}$,

$$
\Phi(F) \subset W \cdot \exp \left(\mathfrak{A}_{\sigma}\right) .
$$

Let $W_{\sigma}$ be the Weyl group of $G_{\sigma}$, that is, the subgroup of $W$ fixing $\exp (\sigma) \subset$ $T$. The connected components of $W \cdot \exp \left(\mathfrak{A}_{\sigma}\right)$ are $W$-translates of $W_{\sigma}$. $\exp \left(\mathfrak{A}_{\sigma}\right)$. Let $w \in W$ be such that $w\left(W_{\sigma} \exp \left(\mathfrak{A}_{\sigma}\right)\right)$ contains $\Phi(F)$. The $\widehat{L G}$ equivariant pre-quantum bundle on $\widehat{M}$ restricts to a pre-quantum bundle for the Hamiltonian $T$-action on $Y_{w \sigma}$, where $T$ is embedded in $\widehat{L G}$ using (16).

Proposition 4.8. The fixed point contribution $\chi\left(\nu_{F}, t\right)$ is related to the fixed point contribution $\chi\left(\nu_{F}^{w \sigma}, t\right)$ for the Hamiltonian T-space $Y^{w \sigma}$ (defined using (3) or (4)) by

$$
\chi\left(\nu_{F}, t\right)=\chi\left(\nu_{F}^{w \sigma}, t\right) \frac{\mathcal{D}_{\mathbb{C}}\left(\mathfrak{g}_{\sigma} / \mathfrak{t}, w^{-1} t\right)}{\mathcal{D}_{\mathbb{C}}\left(\mathfrak{g} / \mathfrak{t}, w^{-1} t\right)} .
$$

In particular, if $\sigma=\{0\}$, we have $\chi\left(\nu_{F}, t\right)=\chi\left(\nu_{F}^{\sigma}, t\right)$. 
Proof. The projection map $\widehat{M} \rightarrow M$ restricts to an equivariant diffeomorphism $\widetilde{F} \cap Y_{w \sigma} \rightarrow F$. Let $\mathcal{L}_{w \sigma}$ be $\operatorname{Spin}_{c}$-line bundle corresponding to $Y_{w \sigma}$. Then

$$
\left.\mathcal{L}_{w \sigma}\right|_{\widetilde{F}} \cong \mathcal{L}_{F} \otimes \mathbb{C}_{2 w\left(\rho-\rho_{\sigma}\right)} .
$$

The normal bundle of $F$ in $M$ splits $T$-equivariantly into the normal bundle $\nu_{F}^{w \sigma}$ in $Y_{w \sigma}$ and the constant bundle $\mathfrak{g} / \mathfrak{g}_{\sigma}$. Using $\mathcal{D}_{\mathbb{R}}\left(\mathfrak{g} / \mathfrak{g}_{\sigma}, t\right) \mathcal{D}_{\mathbb{R}}\left(\mathfrak{g}_{\sigma} / \mathfrak{t}, t\right)=$ $\mathcal{D}_{\mathbb{R}}(\mathfrak{g} / \mathfrak{t}, t)$ we obtain,

$$
\mathcal{D}_{\mathbb{R}}\left(\nu_{F}, t\right)=\mathcal{D}_{\mathbb{R}}\left(\nu_{F}^{w \sigma}, t\right) \frac{\mathcal{D}_{\mathbb{R}}(\mathfrak{g} / \mathfrak{t}, t)}{\mathcal{D}_{\mathbb{R}}\left(\mathfrak{g}_{\sigma} / \mathfrak{t}, t\right)}
$$

Let $\zeta_{F}^{w \sigma}(t)^{1 / 2} \in \mathrm{U}(1)$ be the square root for the action on $\mathcal{L}_{w \sigma}$. We have

$$
\begin{aligned}
\frac{\zeta_{F}(t)^{1 / 2}}{\mathcal{D}_{\mathbb{R}}\left(\nu_{F}, t\right)} & =\frac{\zeta_{F}^{w \sigma}(t)^{1 / 2}}{\mathcal{D}_{\mathbb{R}}\left(\nu_{F}^{w \sigma}, t\right)}(-1)^{\epsilon(w, \sigma)+l(w)} e^{2 \pi i\left\langle w\left(\rho-\rho_{\sigma}\right), v\right\rangle} \frac{\mathcal{D}_{\mathbb{R}}\left(\mathfrak{g}_{\sigma} / \mathfrak{t}, t\right)}{\mathcal{D}_{\mathbb{R}}(\mathfrak{g} / \mathfrak{t}, t)} \\
& =\frac{\zeta_{F}^{w \sigma}(t)^{1 / 2}}{\mathcal{D}_{\mathbb{R}}\left(\nu_{F}^{w \sigma}, t\right)} \frac{\mathcal{D}_{\mathbb{C}}\left(\mathfrak{g}_{\sigma} / \mathfrak{t}, w^{-1} t\right)}{\mathcal{D}_{\mathbb{C}}\left(\mathfrak{g} / \mathfrak{t}, w^{-1} t\right)}
\end{aligned}
$$

\section{Verlinde formula.}

\subsection{The moduli space of flat connections.}

We begin with a brief review of the gauge theory construction of moduli spaces of flat connections. More details can be found in [3], [30], and [14]. Let $\Sigma=\Sigma_{h}^{r}$ denote a compact, connected, oriented surface of genus $h$ with $r$ boundary components. Given a compact, connected, simply-connected Lie group $G$, let $\mathcal{A}(\Sigma)=\underline{\Omega}^{1}(\Sigma, \mathfrak{g})$ be the affine space of connections on the trivial $G$-bundle over $\Sigma$, equipped with the action of $\mathcal{G}(\Sigma)$ by gauge transformations

$$
g \cdot A=\operatorname{Ad}_{g}(A)-g^{*} \theta^{R}
$$

where $\theta^{R}$ is the right-invariant Maurer-Cartan form. Let $\mathcal{G}_{\partial}(\Sigma) \subset \mathcal{G}(\Sigma)$ be the kernel of the restriction map $\mathcal{G}(\Sigma) \rightarrow \mathcal{G}(\partial \Sigma)$. Since $G$ is simply connected, the restriction map is surjective, and therefore $\mathcal{G}(\Sigma) / \mathcal{G}_{\partial}(\Sigma) \cong$ $\mathcal{G}(\partial \Sigma)$. We define

$$
\mathcal{M}(\Sigma):=\mathcal{A}_{\text {flat }}(\Sigma) / \mathcal{G}_{\partial}(\Sigma)
$$


the moduli space of flat $G$-connections under based gauge equivalence. If $\partial \Sigma \neq \emptyset$, it is a smooth $\mathcal{G}(\partial \Sigma)$-equivariant Banach manifold. Pull-back of connections to the boundary induces a map,

$$
\widehat{\Phi}: \mathcal{M}(\Sigma) \rightarrow \underline{\Omega}^{1}(\partial \Sigma, \mathfrak{g}) .
$$

The map $\widehat{\Phi}$ is smooth and proper, and is equivariant for the gauge action of $\mathcal{G}(\partial \Sigma)$. Let $B=B_{k}$ be an invariant inner product on $\mathfrak{g}$, with $k_{j} \in \mathbb{Z}_{>0}$. A symplectic form on $\mathcal{A}(\Sigma)$ is given by the integration pairing of 1 -forms $\left(a_{1}, a_{2}\right) \mapsto \int_{\Sigma} B\left(a_{1} \wedge a_{2}\right)$. As observed by Atiyah-Bott [3], the action of $\mathcal{G}_{\partial}(\Sigma)$ is Hamiltonian, with moment map the curvature. Hence (23) is a symplectic quotient and $\mathcal{M}(\Sigma)$ inherits a symplectic 2-form $\widehat{\omega}$. Moreover, the residual action of $\mathcal{G}(\partial \Sigma)$ on $\mathcal{M}(\Sigma)$ is Hamiltonian with moment map $\widehat{\Phi}$, using the pairing of $\underline{\Omega}^{1}(\partial \Sigma, \mathfrak{g})$ and $\underline{\Omega}^{0}(\partial \Sigma, \mathfrak{g})$ given by the inner product and integration over $\partial \Sigma$. A choice of parametrization of the boundary $\partial \Sigma=$ $\left(S^{1}\right)^{r}$ induces isomorphisms

$$
\mathcal{G}(\partial \Sigma) \cong L G^{r}, \quad \underline{\Omega}^{1}(\partial \Sigma, \mathfrak{g}) \cong \underline{\Omega}^{1}\left(S^{1}, \mathfrak{g}^{r}\right)
$$

Thus $(\mathcal{M}(\Sigma), \widehat{\omega}, \widehat{\Phi})$ is an example of a Hamiltonian $L G^{r}$-manifold with proper moment map. For any $\mu=\left(\mu_{1}, \ldots, \mu_{r}\right) \in L\left(\mathfrak{g}^{r}\right)^{*}$, the symplectic quotient $\mathcal{M}(\Sigma)_{\mu}$ is the moduli space of flat connections for which the holonomy around the $j$ th boundary component is contained in the conjugacy class of $\operatorname{Hol}\left(\mu_{j}\right)$. This also covers the case without boundary, since $M\left(\Sigma_{h}^{0}\right)=M\left(\Sigma_{h}^{1}\right)_{0}$.

Occasionally we will also use the notation $\mathcal{M}(\Sigma, G)$, in order to indicate the structure group. The decomposition into simple factors $G=G_{1} \times \cdots \times G_{s}$ defines a decomposition of the loop group $L G=L G_{1} \times \cdots \times L G_{s}$, and the moduli space is the direct product

$$
\mathcal{M}(\Sigma, G)=\mathcal{M}\left(\Sigma, G_{1}\right) \times \cdots \times \mathcal{M}\left(\Sigma, G_{s}\right)
$$

\subsection{Pre-quantization of the moduli space.}

The space $\mathcal{M}(\Sigma)$ is pre-quantizable at integer level, that is if all $k_{i}$ are integers (see e.g., Section 3.3. of [30] or [17]). For later use, we recall the construction of the pre-quantum line bundle. The central extension $\widehat{\mathcal{G}}(\Sigma)$ of $\mathcal{G}(\Sigma)$ is defined by the cocycle

$$
c\left(g_{1}, g_{2}\right)=\exp \left(i \pi \int_{\Sigma} B\left(g_{1}^{*} \theta^{L}, g_{2}^{*} \theta^{R}\right)\right) .
$$


The group $\widehat{\mathcal{G}}(\Sigma)$ acts on the trivial line bundle over $\mathcal{A}(\Sigma)$ by

$$
(g, z) \cdot(A, w)=\left(g \cdot A, \exp \left(-i \pi \int_{\Sigma} B\left(g^{*} \theta^{L}, A\right)\right) z w\right) .
$$

The 1-form $a \mapsto \frac{1}{2} \int_{\Sigma} B(A, a)$ on $\mathcal{A}(\Sigma)$ defines an invariant pre-quantum connection. A trivialization of $\widehat{\mathcal{G}}(\Sigma)$ over the subgroup $\mathcal{G}_{\partial}(\Sigma)$ is given by the map

$$
\alpha: \mathcal{G}_{\partial}(\Sigma) \rightarrow \mathrm{U}(1), \quad \alpha(g)=\exp \left(2 \pi i \int_{\Sigma \times[0,1]} \bar{g}^{*} \eta\right)
$$

Here $\eta$ is the canonical 3-form on $G$, and $\bar{g} \in \mathcal{G}(\Sigma \times[0,1])$ is any extension such that $\bar{g}=g$ on $\Sigma \times\{0\}$ and $\bar{g}=e$ on $(\Sigma \times\{1\}) \cup(\partial \Sigma \times[0,1])$. The map $\alpha$ is well-defined and satisfies the coboundary condition $\alpha\left(g_{1} g_{2}\right)=$ $\alpha\left(g_{1}\right) \alpha\left(g_{2}\right) c\left(g_{1}, g_{2}\right)$. One defines the pre-quantum line bundle as a quotient $L(\Sigma)=\left(\mathcal{A}_{\text {flat }}(\Sigma) \times \mathbb{C}\right) / \mathcal{G}_{\partial}(\Sigma)$; it comes equipped with an action of $\widehat{L G}=$ $\widehat{\mathcal{G}}(\Sigma) / \mathcal{G}_{\partial}(\Sigma)$.

Our fixed point formula gives a formula for the $\operatorname{Spin}_{c}$-index $\chi\left(\mathcal{M}(\Sigma)_{\mu}\right)$. To apply the fixed point formula we have to (i) describe the holonomy manifold $M(\Sigma):=\mathcal{M}(\Sigma) / \Omega G^{r}$, (ii) determine the fixed point manifolds for elements $\left(t_{\lambda_{1}}, \ldots, t_{\lambda_{r}}\right)$, and (iii) evaluate the fixed point data. These steps will be carried out in the subsequent sections.

\subsection{Holonomy manifolds.}

The holonomy manifold $M(\Sigma):=\mathcal{M}(\Sigma) / \Omega G^{r}$ can be interpreted as the moduli space of flat connections

$$
M(\Sigma)=\mathcal{A}_{\text {flat }}(\Sigma) /\left\{g \in \mathcal{G}(\Sigma) \mid g\left(p_{1}\right)=\ldots=g\left(p_{r}\right)=e\right\}
$$

where $p_{1}, \ldots, p_{r}$ are the base points on the boundary circles. The groupvalued moment map $\Phi: M(\Sigma) \rightarrow G^{r}$ takes an equivalence class of flat connections to its holonomies around the boundary circles. The 2 -form $\omega$ has the following explicit description (see [1, Section 9].) We begin with the case of a 2 -holed sphere $\Sigma_{0}^{2}$. The surface $\Sigma_{0}^{2}$ is obtained from a 4 -gon by identifying the sides according to the word $D_{1} A D_{2} A^{-1}$. Parallel transport along the paths $A$ and $A^{-1} D_{1}$ defines a diffeomorphism

$$
M\left(\Sigma_{0}^{2}\right)=G \times G .
$$


The $G^{2}$-action is given by

$$
\left(g_{1}, g_{2}\right) \cdot(a, b)=\left(g_{1} a g_{2}^{-1}, g_{2} b g_{1}^{-1}\right) .
$$

The moment map is

$$
\Phi(a, b)=\left(a b, a^{-1} b^{-1}\right)
$$

and the 2 -form is given by

$$
\omega=\frac{1}{2}\left(B\left(a^{*} \theta^{L}, b^{*} \theta^{R}\right)+B\left(a^{*} \theta^{R}, b^{*} \theta^{L}\right)\right) .
$$

The holonomy manifolds for the general case $\Sigma=\Sigma_{h}^{r}$ are obtained from $M\left(\Sigma_{0}^{2}\right)$ by fusion, which we recall in Appendix C. First, the moduli space $M\left(\Sigma_{1}^{1}\right)$ for the 1-punctured torus is

$$
M\left(\Sigma_{1}^{1}\right)=M\left(\Sigma_{0}^{2}\right)_{\text {fus }} \cong G^{2} .
$$

The $G$-action is conjugation on each factor and the moment map is the Lie group commutator $\Phi(a, b)=[a, b]=a b a^{-1} b^{-1}$. The moduli space for the surface of genus $h$ with 1 boundary component is an $h$-fold fusion product

$$
M\left(\Sigma_{h}^{1}\right)=M\left(\Sigma_{1}^{1}\right) \circledast \ldots \circledast M\left(\Sigma_{1}^{1}\right)=G^{2 h} .
$$

$G$ acts by conjugation on each factor, and the moment map is a product of Lie group commutators. The moduli space for the $r$-holed sphere $\Sigma_{0}^{r}$ is an $(r-1)$-fold fusion product

$$
M\left(\Sigma_{0}^{r}\right)=M\left(\Sigma_{0}^{2}\right) \circledast \ldots \circledast M\left(\Sigma_{0}^{2}\right)=G^{2(r-1)}
$$

where we fuse with respect to the first $G$-factor for each $G^{2}$-space $M\left(\Sigma_{0}^{2}\right)$. Finally, the moduli space for $\Sigma_{h}^{r}$ is

$$
M\left(\Sigma_{h}^{r}\right)=M\left(\Sigma_{h}^{1}\right) \circledast M\left(\Sigma_{0}^{r}\right)=G^{2(h+r-1)} .
$$

\subsection{The fixed point sets.}

The fixed point sets for the action on the holonomy manifold are symplectic tori:

Proposition 5.1. The fixed point set for the action of $\left(t_{\lambda_{1}}, \ldots, t_{\lambda_{r}}\right)$ on $M\left(\Sigma_{h}^{r}\right)=G^{2(h+r-1)}$ is empty unless $\lambda_{1}=\ldots=\lambda_{r}=: \lambda$, and

$$
M\left(\Sigma_{h}^{r}\right)^{\left(t_{\lambda}, \ldots, t_{\lambda}\right)}=F:=T^{2(h+r-1)} .
$$


Proof. Since $M\left(\Sigma_{h}^{r}\right)$ is obtained from a direct product of $h+r-1$ copies of $M\left(\Sigma_{0}^{2}\right)$ by passing to diagonal actions for some of the $G$-factors, it suffices to prove Proposition 5.1 for $\Sigma_{0}^{2}$. By (27), an element $(a, b) \in M\left(\Sigma_{0}^{2}\right)$ is fixed by $\left(t_{\lambda_{1}}, t_{\lambda_{2}}\right)$ if and only if

$$
t_{\lambda_{1}}=\operatorname{Ad}_{a} t_{\lambda_{2}}, \quad t_{\lambda_{2}}=\operatorname{Ad}_{b} t_{\lambda_{1}}
$$

Both $t_{\lambda_{1}}$ and $t_{\lambda_{2}}$ belong to the exponential of the alcove $\exp (\mathfrak{A})$. Since each conjugacy class meet $\exp (\mathfrak{A})$ only once, (30) holds if and only if $\lambda_{1}=\lambda_{2}$.

Notice that the fixed point set is independent of $\lambda$; in fact, $F$ is fixed by the full diagonal torus $T \subset G^{r}$.

\subsection{Evaluation of the fixed point contributions.}

Let $\Sigma=\Sigma_{h}^{r}$ and $\mu=\left(\mu_{1}, \ldots, \mu_{r}\right)$ with $\mu_{j} \in \Lambda_{k}^{*}$. By Theorem 4.3, the Spin $_{c}$-index is given by the formula

$$
\chi\left(\mathcal{M}(\Sigma)_{\mu}\right)=\frac{1}{\left(\# T_{k+c}\right)^{r}} \sum_{\lambda \in \Lambda_{k}^{*}} \chi_{\mu_{1}}\left(t_{\lambda}\right) \cdots \chi_{\mu_{r}}\left(t_{\lambda}\right)\left|J\left(t_{\lambda}\right)\right|^{2 r} \chi\left(\nu_{F}, t_{\lambda}\right)^{*}
$$

Here we abbreviated $\left(t_{\lambda}, \ldots, t_{\lambda}\right)$ to $t_{\lambda}$, viewing $T$ as diagonally embedded into $G^{r}$. For the calculation of the fixed point contribution

$$
\chi\left(\nu_{F}, t_{\lambda}\right)=\zeta_{F}\left(t_{\lambda}\right)^{1 / 2} \int_{F} \frac{\hat{A}(F) e^{\frac{1}{2} c_{1}\left(\mathcal{L}_{F}\right)}}{\mathcal{D}_{\mathbb{R}}\left(\nu_{F}, t_{\lambda}\right)}
$$

note first of all that

$$
\hat{A}(F)=1
$$

since $F$ is a torus. Furthermore, since the normal bundle $\nu_{F}$ is $T$ equivariantly isomorphic to $(\mathfrak{g} / \mathfrak{t})^{2(h+r-1)}$, we have

$$
\mathcal{D}_{\mathbb{R}}\left(\nu_{F}, t_{\lambda}\right)=J\left(t_{\lambda}\right)^{2(h+r-1)}=(-1)^{(h+r-1) \# \Re}\left|J\left(t_{\lambda}\right)\right|^{2(h+r-1)} .
$$

It remains to work out the integral $\int_{F} \exp \left(\frac{1}{2} c_{1}\left(\mathcal{L}_{F}\right)\right)$ and to calculate the phase factor $\zeta_{F}\left(t_{\lambda}\right)^{1 / 2}$.

Proposition 5.2. The integral of $\exp \left(\frac{1}{2} c_{1}\left(\mathcal{L}_{F}\right)\right)$ over $F$ equals $\left(\# T_{k+c}\right)^{h+r-1}$. 
Proof. The line bundle $\mathcal{L}=L(\Sigma)^{2} \otimes K^{-1}$ is $\widehat{L G^{r}}$-equivariant at levels $2(k+c), \ldots, 2(k+c)$. Since $\mathcal{M}(\Sigma)$ carries up to isomorphism a unique line bundle at every level $[29,3.12]$, it follows that $\mathcal{L}$ is the pre-quantum line bundle for the symplectic structure defined by $B_{2(k+c)}$. Hence $\mathcal{L}_{F}$ is a pre-quantum line bundle for the corresponding symplectic structure on $F$ (cf. [?], Subsection 4.4.3), and $\int_{F} \exp \left(\frac{1}{2} c_{1}\left(\mathcal{L}_{F}\right)\right)$ is the symplectic volume $\operatorname{Vol}_{B_{k+c}}(F)$ for the 2-form defined using $B_{k+c}$. We claim that the symplectic volume coincides with the Riemannian volume, which will complete the proof since $\operatorname{Vol}_{B_{k+c}}\left(T^{2}\right)=\# T_{k+c}$ (see e.g., Beauville [8, Remark 9.9], Bismut-Labourie [10, Prop. 1.2, 1.3]). By our description of $M\left(\Sigma_{h}^{r}\right)$ as a fusion product, the fixed point manifold $F=F\left(\Sigma_{h}^{r}\right)$ is obtained from the fixed point manifold $F\left(\Sigma_{0}^{2}\right)$ (viewed as a group valued Hamiltonian $T^{2}$-space) by fusion: $F\left(\Sigma_{1}^{1}\right)=F\left(\Sigma_{0}^{2}\right)_{\text {fus }}$ and

$$
F\left(\Sigma_{h}^{r}\right)=F\left(\Sigma_{1}^{1}\right) \circledast \cdots \circledast F\left(\Sigma_{1}^{1}\right) \circledast F\left(\Sigma_{0}^{2}\right) \circledast \cdots \circledast F\left(\Sigma_{0}^{2}\right),
$$

with $h$ factors $F\left(\Sigma_{1}^{1}\right)$ and $(r-1)$ factors $F\left(\Sigma_{0}^{2}\right)$. Lemma C.2 from Appendix $\mathrm{C}$ says that the symplectic volume of group valued Hamiltonian torus spaces does not change under fusion. Hence $\operatorname{Vol}_{B_{k+c}}\left(F\left(\Sigma_{h}^{r}\right)\right)=$ $\operatorname{Vol}_{B_{k+c}}\left(F\left(\Sigma_{0}^{2}\right)\right)^{h+r-1}$. Finally, the expression (29) for the 2-form on $M\left(\Sigma_{0}^{2}\right)$ shows that $\operatorname{Vol}_{B_{k+c}}\left(F\left(\Sigma_{0}^{2}\right)\right)$ coincides with the Riemannian volume of $T^{2}$ with respect to $B_{k+c}$.

Proposition 5.3. The phase factor is given by $\zeta_{F}\left(t_{\lambda}\right)^{1 / 2}=(-1)^{(h+r-1) \# \Re_{+}}$.

Proof. The point $m=(e, \ldots, e) \in F$ lies in identity level set of $\Phi$, and its stabilizer in $G^{r}$ is the image of the diagonal embedding of $G$. The 2form $\omega$ restricts to a symplectic form on the tangent space $E=T_{m} M(\Sigma)$. By Equations (27) and (29) of [?], $\zeta_{F}\left(t_{\lambda}\right)^{1 / 2}$ can be computed in terms of the symplectomorphism $A$ of $E$ defined by $t_{\lambda}$ : Choose an $A$-invariant compatible complex structure on $E$ to view $A$ as a unitary transformation, and let $A^{1 / 2}$ be the unique square root having all its eigenvalues in the set $\left\{e^{i \phi} \mid 0 \leq \phi<\pi\right\}$. Then $\zeta_{F}\left(t_{\lambda}\right)^{1 / 2}=\operatorname{det}\left(A^{1 / 2}\right)$.

We first apply this recipe for the 2-holed sphere $\Sigma_{0}^{2}$, so that $E=$ $T_{m} M\left(\Sigma_{0}^{2}\right)=\mathfrak{g} \oplus \mathfrak{g}$. Formula (29) shows that $\omega_{m}$ is the standard 2-form on $\mathfrak{g} \oplus \mathfrak{g}$, given by the inner product $B$. A compatible complex structure is given by the endomorphism $(\xi, \eta) \mapsto(-\eta, \xi)$. Thus, as a complex $G$ representation $E$ is just the complexification $E=\mathfrak{g}^{\mathbb{C}}$. It follows that the eigenvalues of $A$ (other than 1) come in complex conjugate pairs

$$
e^{i \phi_{j}}, e^{-i \phi_{j}}, \quad 0<\phi_{j} \leq \pi / 2
$$


and the corresponding eigenvalues of $A^{1 / 2}$ are $e^{i \phi_{j} / 2}$ and $e^{i \pi-i \phi_{j} / 2}=$ $-e^{-i \phi_{j} / 2}$. Hence

$$
\zeta_{F}\left(t_{\lambda}\right)^{1 / 2}=(-1)^{\# \Re_{+}} .
$$

Now consider the case $r \geq 1, h$ arbitrary. The tangent space is $T_{m} M\left(\Sigma_{h}^{r}\right)=$ $(\mathfrak{g} \oplus \mathfrak{g})^{h+r-1}$, but because of the fusion terms the symplectic form is not the standard symplectic form defined by the inner product on $\mathfrak{g}$. However, by Appendix C, Lemma C.3 it is equivariantly and symplectically isotopic to the standard symplectic form. Since the phase factor $\zeta_{F}\left(t_{\lambda}\right)^{1 / 2}$ is a root of unity, it is invariant under equivariant symplectic isotopies, and we conclude as before that $\zeta_{F}\left(t_{\lambda}\right)^{1 / 2}=(-1)^{(h+r-1) \# \mathfrak{R}_{+}}$.

We conclude that the fixed point integral is given by

$$
\chi\left(\nu_{F}, t_{\lambda}\right)=\left(\frac{\# T_{k+c}}{\left|J\left(t_{\lambda}\right)\right|^{2}}\right)^{h+r-1}
$$

\subsection{Verlinde formula.}

From (34) and (31) we obtain:

Theorem 5.4 (Verlinde Formula). Let $G$ be a simply connected Lie group and $k$ a given integral level. The $\operatorname{Spin}_{c}$-index of the moduli space of flat connections on $\Sigma_{h}^{r}$ at level $k$, with markings $\mu=\left(\mu_{1}, \ldots, \mu_{r}\right) \in\left(\Lambda_{k}^{*}\right)^{r}$ is given by the formula

$$
\chi\left(\mathcal{M}\left(\Sigma_{h}^{r}\right)_{\mu}\right)=\left(\# T_{k+c}\right)^{h-1} \sum_{\lambda \in \Lambda_{k}^{*}}\left|J\left(t_{\lambda}\right)\right|^{2-2 h} \chi_{\mu_{1}}\left(t_{\lambda}\right) \cdots \chi_{\mu_{r}}\left(t_{\lambda}\right) .
$$

Remarks 5.5. (a) Theorem (5.4) also covers the case without boundary, since $M\left(\Sigma_{h}^{0}\right)=M\left(\Sigma_{h}^{1}, 0\right)$. One obtains

$$
\chi\left(\mathcal{M}\left(\Sigma_{h}^{0}\right)\right)=\left(\# T_{k+c}\right)^{h-1} \sum_{\lambda \in \Lambda_{k}^{*}}\left|J\left(t_{\lambda}\right)\right|^{2-2 h} .
$$

(b) For the two-holed sphere $\Sigma_{0}^{2}$, formula (35) simplifies by the orthogonality relations for level $k$ characters, and gives $\chi\left(M\left(\Sigma_{0}^{2}\right)_{\mu_{1}, \mu_{2}}\right)=\delta_{\mu_{1}, * \mu_{2}}$.

(c) In Bismut-Labourie [10] the $\operatorname{Spin}_{c}$-indices $\chi\left(\mathcal{M}\left(\Sigma_{h}^{r}\right)_{\mu}\right)$ are computed by direct application of the Kawasaki-Riemann-Roch formula to the reduced spaces. Their approach involves a description of all orbifold strata of the reduced space. The equality with the above sum over 
level $k$ weights is non-trivial; it is established in [10] for sufficiently high level $k$.

(d) Theorem 5.4 gives a formula for a $\mathrm{Spin}_{c^{-}}$index rather than the dimension of a space of holomorphic sections. Vanishing results for higher cohomology groups have recently been proved by Teleman [34, Section $8]$.

(e) It is possible to extend our approach to arbitrary semi-simple, compact, connected groups which need not be simply connected. The calculation is more involved since there are more fixed point components in general. The non-simply connected case will be discussed in a sequel to this paper.

\section{Proof of the fixed point formula.}

Our proof of the fixed point formula proceeds in two stages. First, we show that in case $\widehat{M}$ admits a global cross-section, the formula follows from the "quantization commutes with reduction theorem" applied to the crosssection. In a second step we reduce to this case using the method of symplectic cutting.

\subsection{An identity for level $k$ characters.}

Let $G$ be equipped with inner product $B=B_{k}$ where $k \in\left(\mathbb{Z}_{>0}\right)^{s}$. We will need a Lemma expressing the restrictions of irreducible level $k$ characters of $G$ to the group $T_{k+c}$, in terms of characters of central extensions $\widehat{G}_{\sigma}$ of $G_{\sigma}$ obtained as the pull-back of $\widehat{L G}^{(k)}$ by the map $G_{\sigma} \cong L G_{\sigma}$ (cf. (9)). For $\sigma \subset \bar{\tau}$ we have embeddings $\widehat{G}_{\tau} \subset \widehat{G}_{\sigma}$, in particular every $\widehat{G}_{\sigma}$ contains $\widehat{T}$ as a maximal torus. Let $\widehat{T}=T \times \mathrm{U}(1)$ be the trivialization obtained by restricting the trivialization (16). In terms of the corresponding splitting $\widehat{\mathfrak{t}}^{*}=\mathfrak{t}^{*} \times \mathbb{R}$, the action of $W_{\sigma}$ on $\widehat{\mathfrak{t}}^{*}$ reads

$$
w_{1} \cdot(\mu, \tau)=\left(w_{1} \mu+\tau B^{b}\left(\gamma_{\sigma}-w_{1} \gamma_{\sigma}\right), \tau\right)
$$

and a positive Weyl chamber for $\widehat{G}_{\sigma}$ is given by

$$
\widehat{\mathfrak{t}}_{\sigma,+}^{*}=\left(\mathfrak{t}_{\sigma,+}^{*} \times\{0\}\right)+\mathbb{R} \cdot\left(B^{b}\left(\gamma_{\sigma}\right), 1\right) .
$$

For any level $k$ weight $\mu \in \Lambda_{k}^{*}$, the weight $(\mu, 1) \in \Lambda^{*} \times \mathbb{Z}$ is contained in the positive Weyl chamber for $\widehat{G}_{\sigma}$, and hence parametrizes an irreducible 
representation. Consider the restriction of its character $\chi_{\mu, \sigma} \in C^{\infty}\left(\widehat{G}_{\sigma}\right)$ to

$$
T_{k+c} \subset T \subset \widehat{T} \subseteq \widehat{G}_{\sigma}
$$

We identify $W / W_{\sigma}$ with the set of all $w \in W$ such that $w\left(\mathfrak{t}_{+}\right) \subset \mathfrak{t}_{+, \sigma}$. Every element in $W$ can be uniquely written in the form $w w_{1}$ with $w \in W / W_{\sigma}$ and $w_{1} \in W_{\sigma}$.

Lemma 6.1. For all $t \in T_{k+c}$, and all $\mu \in \Lambda_{k}^{*}$,

$$
\chi_{\mu}(t)=\sum_{w \in W / W_{\sigma}} \chi_{\mu, \sigma}\left(w^{-1} t\right) \frac{\mathcal{D}_{\mathbb{C}}\left(\mathfrak{g}_{\sigma} / \mathfrak{t}, w^{-1} t\right)}{\mathcal{D}_{\mathbb{C}}\left(\mathfrak{g} / \mathfrak{t}, w^{-1} t\right)}
$$

Proof. By the Weyl character formula,

$$
\begin{aligned}
\chi_{\mu}(t) & =\sum_{w \in W / W_{\sigma}} \sum_{w_{1} \in W_{\sigma}}(-1)^{l\left(w w_{1}\right)} \frac{t^{w w_{1}(\mu+\rho)-\rho}}{\mathcal{D}_{\mathbb{C}}(\mathfrak{g} / \mathfrak{t}, t)} \\
& =\sum_{w \in W / W_{\sigma}} \frac{\sum_{w_{1} \in W_{\sigma}}(-1)^{l\left(w_{1}\right)}\left(w^{-1} t\right)^{w_{1}(\mu+\rho)-\rho}}{\mathcal{D}_{\mathbb{C}}\left(\mathfrak{g} / \mathfrak{t}, w^{-1} t\right)} .
\end{aligned}
$$

Given $t \in W / W_{\sigma}$ let $t_{1}=w^{-1} t$. We claim that the sum over $W_{\sigma}$ is just

$$
\mathcal{D}_{\mathbb{C}}\left(\mathfrak{g}_{\sigma} / \mathfrak{t}, t_{1}\right) \chi_{\mu, \sigma}\left(t_{1}\right)=\sum_{w_{1} \in W_{\sigma}}(-1)^{l\left(w_{1}\right)} t_{1}^{w_{1}\left(\mu+\rho_{\sigma}, 1\right)-\left(\rho_{\sigma}, 0\right)} .
$$

Indeed, by (36) and since $\rho_{\sigma}=\rho-B_{c}^{b}\left(\gamma_{\sigma}\right)$, we have

$$
w_{1}\left(\mu+\rho_{\sigma}, 1\right)-\left(\rho_{\sigma}, 0\right)=\left(w_{1}(\mu+\rho)-\rho+B_{k+c}^{b}\left(\gamma_{\sigma}-w_{1} \gamma_{\sigma}\right), 1\right) .
$$

But $t_{1}^{B_{k+c}^{b}\left(\gamma_{\sigma}-w_{1} \gamma_{\sigma}\right)}=1$ since $t_{1} \in T_{k+c}$. Hence $t_{1}^{w_{1}\left(\mu+\rho_{\sigma}, 1\right)-\left(\rho_{\sigma}, 0\right)}=$ $t_{1}^{w_{1}(\mu+\rho)-\rho}$, proving the claim.

\subsection{Proof in case of a global cross-section.}

We now explain the proof of Theorem 4.3 in the special case where $(\widehat{M}, \widehat{\omega}, \widehat{\Phi})$ admits a global cross-section. That is, we make the assumption that for some face $\sigma$ of the alcove, the moment polytope is contained in $\mathfrak{A}_{\sigma}$. As a consequence

$$
\widehat{M}=L G \times_{L G_{\sigma}} Y_{\sigma} .
$$


Using the identification $\widehat{L G}_{\sigma} \cong \widehat{G}_{\sigma}$, we view $Y_{\sigma}$ as a Hamiltonian $\widehat{G}_{\sigma}$-space. Clearly, $\widehat{M}_{\mu}=\left(Y_{\sigma}\right)_{\mu}$ for all $\mu \in \Lambda_{k}^{*}$. Using Lemma 6.1 and the "quantization commutes with reduction" principle (Theorem 2.1),

$$
\begin{aligned}
\sum_{\mu \in \Lambda_{k}^{*}} \chi\left(\widehat{M}_{\mu}\right) \chi_{\mu}(t) & =\sum_{\mu \in \Lambda_{k}^{*}} \chi\left(\left(Y_{\sigma}\right)_{\mu}\right) \sum_{w \in W / W_{\sigma}} \chi_{\mu, \sigma}\left(w^{-1} t\right) \frac{\mathcal{D}_{\mathbb{C}}\left(\mathfrak{g}_{\sigma} / \mathfrak{t}, w^{-1} t\right)}{\mathcal{D}_{\mathbb{C}}\left(\mathfrak{g} / \mathfrak{t}, w^{-1} t\right)} \\
& =\sum_{w \in W / W_{\sigma}} \chi\left(Y_{\sigma}, w^{-1} t\right) \frac{\mathcal{D}_{\mathbb{C}}\left(\mathfrak{g}_{\sigma} / \mathfrak{t}, w^{-1} t\right)}{\mathcal{D}_{\mathbb{C}}\left(\mathfrak{g} / \mathfrak{t}, w^{-1} t\right)}
\end{aligned}
$$

Theorem 4.3 now follows by an application of the fixed point formula to $Y_{\sigma}$, and using Proposition 4.8 for the fixed point contributions.

\subsection{Proof in the general case.}

Our proof of Theorem 4.3 in the general case is an application of symplectic cutting, reviewed in Appendix B.

Denote by $\Psi: M \rightarrow \mathfrak{A}$ the composition of the map $\Phi: M \rightarrow G$ with the quotient map $G \rightarrow G / \operatorname{Ad}(G)=\mathfrak{A}$. For suitable polytopes $Q \subset \mathfrak{t}$, the cut spaces $M_{Q}$ will be obtained by collapsing the boundary of $\Psi^{-1}(Q)$ in a certain way. The polytopes $Q$ are defined as follows.

Pick a rational point $\mu \in \Lambda \otimes_{\mathbb{Z}} \mathbb{Q}$ in the interior of the alcove, and let $\epsilon \in \mathbb{Q}$ with $0<\epsilon<1$. For any face $\sigma$ of $\mathfrak{A}$, let $Q=Q_{\sigma}$ be the convex hull of all conjugates of $(1-\epsilon) \bar{\sigma}+\epsilon \mu \subset \mathfrak{A}$ under the affine action of $W_{\sigma}$. (See Figure 1). The polytope $Q$ is simplicial; choose integral labels as in B.1. Notice that $Q \cap \mathfrak{A} \subset \mathfrak{A}_{\tau}$ for all $\tau \subseteq \bar{\sigma}$.

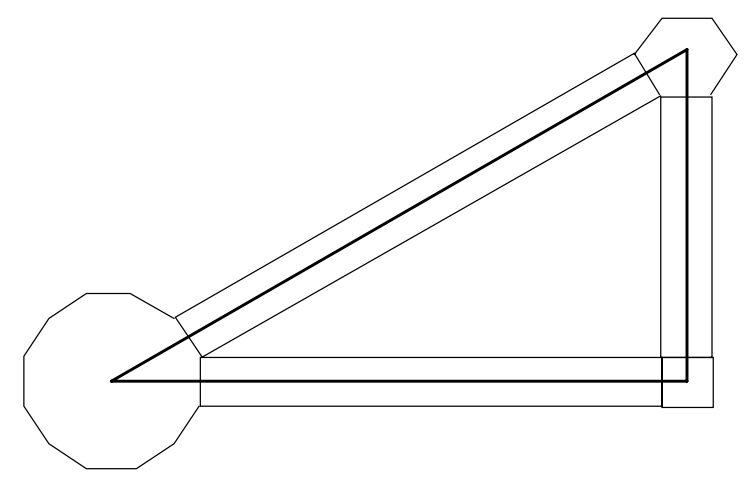

Figure 1: The polytopes $Q_{\sigma}$ for $G_{2}$. The bold-faced line indicates the boundary of the Weyl alcove $\mathfrak{A}$. 
The polytope $Q=Q_{\sigma}$ will be called $\Phi$-admissible if it is $\widehat{\Phi}_{\sigma}$-admissible (cf. Appendix B.2) for $Y_{\sigma}$. It is then also $\widehat{\Phi}_{\tau^{-}}$admissible for $Y_{\tau}$ for each $\tau \subseteq \bar{\sigma}$. The cut spaces satisfy $\left(Y_{\sigma}\right)_{Q}=G_{\sigma} \times_{G_{\tau}}\left(Y_{\tau}\right)_{Q}$, so that the orbifold $M_{Q}:=$ $G \times{ }_{G_{\tau}}\left(Y_{\tau}\right)_{Q}$ is independent of the choice of $\tau$ with $\tau \subseteq \bar{\sigma}$. There is a natural map $\Psi^{-1}(Q) \rightarrow M_{Q}$ which is a diffeomorphism over $\Psi^{-1}(\operatorname{int}(Q))$. More generally, for $Q$ a face of $Q_{\sigma}$ we let $\left(Y_{\tau}\right)_{Q}$ be the corresponding symplectic sub-orbifold of $\left(Y_{\tau}\right)_{Q_{\sigma}}$, and $M_{Q}:=G \times_{G_{\tau}}\left(Y_{\tau}\right)_{Q}$ is a sub-orbifold of $M_{Q_{\sigma}}$. Guided by Lemma 6.1 we define, for $t \in T_{k+c}$,

$$
\chi\left(M_{Q}, t\right):=\sum_{w \in W / W_{\tau}} \frac{\mathcal{D}_{\mathbb{C}}\left(\mathfrak{g}_{\tau} / \mathfrak{t}, w^{-1} t\right)}{\mathcal{D}_{\mathbb{C}}\left(\mathfrak{g} / \mathfrak{t}, w^{-1} t\right)} \chi\left(\left(Y_{\tau}\right)_{Q}, w^{-1} t\right),
$$

which again is independent of the choice of $\tau$.

Let $\mathcal{Q}$ be the collection of all $Q_{\sigma}$, along with their conjugates under the action of the affine Weyl group $W_{\text {aff }}$. By a generic choice of $\mu, \epsilon$, we can assume that all $Q=Q_{\sigma}$ are $\Phi$-admissible. Let $\widetilde{\mathcal{Q}}$ be the set of all polytopes $Q \in \mathcal{Q}$, and all of their closed faces. The following observation is our starting point for the proof of Theorem 4.3.

Lemma 6.2. For all $t \in T_{k+c}^{\mathrm{reg}}$,

$$
\chi(M, t)=\sum_{Q \in \widetilde{\mathcal{Q}}}(-1)^{\operatorname{codim} Q} \chi\left(M_{Q}, t\right) .
$$

Proof. For all $\mu \in \Lambda_{k}^{*} \cap Q$, with $Q \cap \mathfrak{A} \subset \mathfrak{A}_{\sigma}$ we have $\left(\left(Y_{\sigma}\right)_{Q}\right)_{\mu}=\widehat{M}_{\mu}$. Hence, "quantization commutes with reduction" (Theorem 2.1) together with Lemma 6.1 shows that

$$
\chi\left(M_{Q}, t\right)=\sum_{\mu \in \Lambda_{k}^{*} \cap Q} \chi\left(\widehat{M}_{\mu}\right) \chi_{\mu}(t) .
$$

Let $1_{Q}$ be the characteristic function of $Q$. Using the Euler formula

$$
\sum_{Q \in \tilde{\mathcal{Q}}}(-1)^{\operatorname{codim} Q_{1}} 1_{Q}(\mu)=1
$$

the alternating sum over $\chi\left(M_{Q}, t\right)$ equals $\sum_{\mu \in \Lambda_{k}^{*}} \chi\left(\widehat{M}_{\mu}\right) \chi_{\mu}(t)$.

The orbifold version of the fixed point formula, Theorem A.2 in Appendix A, expresses all indices $\chi\left(\left(Y_{\sigma}\right)_{Q}, t\right)$, and therefore all $\chi\left(M_{Q}, t\right)$, as a sum 
over fixed point contributions. Our aim is to identify this sum with the sum over fixed point contributions $\sum_{F \subseteq M^{t}} \chi\left(\nu_{F}, t\right)$. To obtain the required gluing formula, we would like to localize further to the fixed point set of the maximal torus $T \subset G$. However, a problem arises because the $T_{2(k+c)}$-action on $\mathcal{L}_{F} \rightarrow F$ need not extend to a $T$-action. Over each $F \cap Y_{\sigma}$ such a $T$-action can be introduced by choice of a moment map, however the local $T$-actions obtained in this way do not fit together in general.

In order to get around this problem, we proceed as in [30, Appendix A] and consider a second collection $\mathcal{S}=\{S\}$ of integral labeled polytopes in $\mathfrak{t}$. The polytopes in $\mathcal{S}$ are constructed just like those in $\mathcal{Q}$, but with $\epsilon$ replaced by some $\epsilon^{\prime}>\epsilon$. By a generic choice of $\mu, \epsilon, \epsilon^{\prime}$ we may assume that all $S \in \mathcal{S}$ and all intersections $S \cap Q$ with $S \in \mathcal{S}$ and $Q \in \mathcal{Q}$ are admissible. Given $S \in \mathcal{S}$ and $t \in T_{k+c}^{\mathrm{reg}}$, we define

$$
\chi_{S}(M, t)=\sum_{F \subseteq M^{t}} \chi_{S}\left(\nu_{F}, t\right)
$$

where $\chi_{S}\left(\nu_{F}, t\right)$ is defined by an integral similar to $\chi\left(\nu_{F}, t\right)$ (cf. (18)), but integrating only over the subset $F \cap \Psi^{-1}(S)$ :

$$
\chi_{S}\left(\nu_{F}, t\right):=\zeta_{F}(t)^{1 / 2} \int_{F \cap \Psi^{-1}(S)} \frac{\widehat{A}(F) e^{\frac{1}{2} c_{1}\left(\mathcal{L}_{F}\right)}}{\mathcal{D}_{\mathbb{R}}\left(\nu_{F}, t\right)} .
$$

Lemma 6.3. For all $S \in \mathcal{S}$, the integral $\chi_{S}\left(\nu_{F}, t\right)$ is independent of the choices of differential form representatives $\widehat{A}(F), c_{1}\left(\mathcal{L}_{F}\right)$ and $\mathcal{D}_{\mathbb{R}}\left(\nu_{F}, t\right)$, provided these are chosen in such a way that for each boundary face $R \subset S$, the pull-back of the form to $F \cap \Psi^{-1}(R)$ is $T_{R}$-basic. We have

$$
\chi\left(\nu_{F}, t\right)=\sum_{S \in \mathcal{S}} \chi_{S}\left(\nu_{F}, t\right) .
$$

Proof. The first part follows by observing that the integral can be re-written as an integral over the cut space $F_{S} \subset M_{S}$ of $F$, that is over the image of $F \cap \Psi^{-1}(S)$ in $M_{S}=\Psi^{-1}(S) / \sim$ :

$$
\begin{aligned}
\chi_{S}\left(\nu_{F}, t\right) & =\zeta_{F}(t)^{1 / 2} \int_{F \cap \Psi^{-1}(S)} \frac{\widehat{A}(F) e^{\frac{1}{2} c_{1}\left(\mathcal{L}_{F}\right)}}{\mathcal{D}_{\mathbb{R}}\left(\nu_{F}, t\right)} \\
& =\zeta_{F}(t)^{1 / 2} \int_{F_{S}} \frac{\widehat{A}\left((T F)_{S}\right) e^{\frac{1}{2} c_{1}\left(\left(\mathcal{L}_{F}\right)_{S}\right)}}{\mathcal{D}_{\mathbb{R}}\left(\left(\nu_{F}\right)_{S}, t\right)} .
\end{aligned}
$$


Here $(T F)_{S}=\left.T F\right|_{F \cap \Psi^{-1}(S)} / \sim$ is the "cut" of $T F$ as explained in Appendix B.2, and similarly for $\left(\nu_{F}\right)_{S}$ and $\left(\mathcal{L}_{F}\right)_{S}$. Formula (38) is expressing the integral over $F$ as a sum of integrals over all pieces $F \cap \Psi^{-1}(S)$ in the decomposition.

The integrals $\chi_{S}\left(\nu_{F}, t\right)$ can be re-written in terms of cross-sections $Y_{\sigma}$. As above, we identify $Y_{\sigma} \subset \widehat{M}$ with its image under the map $\widehat{M} \rightarrow M$, and interpret $Y_{\sigma}$ as a Hamiltonian $\widehat{G}_{\sigma} \cong \widehat{L G}_{\sigma}$-space. Let $\Psi_{\sigma}: Y_{\sigma} \rightarrow \mathfrak{A}$ be the restriction of $\Psi$. It can be identified with the composition of the moment map $\widehat{\Phi}_{\sigma}$ with the projection map $\widehat{\mathfrak{g}}_{\sigma}^{*} \rightarrow \widehat{\mathfrak{t}}_{+}^{*} \supset \mathfrak{A} \times\{1\}$. The intersection $Y_{\sigma} \cap \Psi_{\sigma}^{-1}(S)$ is compact, and we can define

$$
\chi_{S}\left(Y_{\sigma}, t\right)=\sum_{F \subseteq\left(Y_{\sigma}\right)^{t}} \chi_{S}\left(\nu_{F}^{\sigma}, t\right)
$$

with

$$
\chi_{S}\left(\nu_{F}^{\sigma}, t\right)=\mu_{F}(t) \int_{F \cap \Psi_{\sigma}^{-1}(S)} \frac{\operatorname{Td}(F) e^{c_{1}\left(\left.L_{\sigma}\right|_{F}\right)}}{\mathcal{D}_{\mathbb{C}}\left(\nu_{F}^{\sigma}, t\right)},
$$

where $\nu_{F}^{\sigma}$ is the normal bundle of $F$ in $Y_{\sigma}$. As in Lemma 6.3, the integral does not depend on representatives for $\operatorname{Td}(F), c_{1}\left(\left.L_{\sigma}\right|_{F}\right)$ and $\mathcal{D}_{\mathbb{C}}\left(\nu_{F}^{\sigma}, t\right)$, provided for each open face $R \subset S$ the pull-backs to $\Psi_{\sigma}^{-1}(R) \cap F$ descend to the quotient by $T_{R}$. Following the argument in Section 4.4, we have

$$
\chi_{S}\left(\nu_{F}, t\right)=\sum_{w \in W / W_{\sigma}} \frac{\mathcal{D}_{\mathbb{C}}\left(\mathfrak{g}_{\sigma} / \mathfrak{t}, w^{-1} t\right)}{\mathcal{D}_{\mathbb{C}}\left(\mathfrak{g} / \mathfrak{t}, w^{-1} t\right)} \chi_{S}\left(\nu_{F}^{\sigma}, w^{-1} t\right),
$$

hence

$$
\chi_{S}(M, t)=\sum_{w \in W / W_{\sigma}} \frac{\mathcal{D}_{\mathbb{C}}\left(\mathfrak{g}_{\sigma} / \mathfrak{t}, w^{-1} t\right)}{\mathcal{D}_{\mathbb{C}}\left(\mathfrak{g} / \mathfrak{t}, w^{-1} t\right)} \chi_{S}\left(Y_{\sigma}, w^{-1} t\right) .
$$

Over $Y_{\sigma}$, we have a Hamiltonian $T$-action with $T$-equivariant pre-quantum line bundle. Hence $\chi_{S}\left(Y_{\sigma}, w^{-1} t\right)$ can be written as a limit of $\chi_{S}\left(Y_{\sigma}, w^{-1}(t \exp \xi)\right)$ as $\xi \in \mathfrak{t}$ approaches 0 , and by the Berline-Vergne formula the integral defining $\chi_{S}\left(Y_{\sigma}, w^{-1}(t \exp \xi)\right)$ localizes to the fixed point set of $T$. The details of this approach are given in Appendix B. In particular Proposition B. 1 allows us to re-write $\chi_{S}\left(Y_{\sigma}, w^{-1} t\right)$ as an alternating sum over the corresponding terms for the cut spaces $\left(Y_{\sigma}\right)_{Q}$ :

$$
\chi_{S}\left(Y_{\sigma}, w^{-1} t\right)=\sum_{Q \in \tilde{\mathcal{Q}}}(-1)^{\operatorname{codim} Q} \chi_{S}\left(\left(Y_{\sigma}\right)_{Q}, w^{-1} t\right) .
$$


From (39) we obtain,

$$
\begin{aligned}
& \sum_{F \subseteq M^{t}} \chi\left(\nu_{F}, t\right)=\sum_{S \in \mathcal{S}} \chi_{S}(M, t) \\
& =\sum_{S \in \mathcal{S}} \sum_{Q \in \tilde{\mathcal{Q}}}(-1)^{\operatorname{codim} Q}\left(\sum_{w \in W / W_{\sigma}} \frac{\mathcal{D}_{\mathbb{C}}\left(\mathfrak{g}_{\sigma} / \mathfrak{t}, w^{-1} t\right)}{\mathcal{D}_{\mathbb{C}}\left(\mathfrak{g} / \mathfrak{t}, w^{-1} t\right)} \chi_{S}\left(\left(Y_{\sigma}\right)_{Q}, w^{-1} t\right)\right) \\
& =\sum_{Q \in \tilde{\mathcal{Q}}}(-1)^{\operatorname{codim} Q}\left(\sum_{w \in W / W_{\sigma}} \frac{\mathcal{D}_{\mathbb{C}}\left(\mathfrak{g}_{\sigma} / \mathfrak{t}, w^{-1} t\right)}{\mathcal{D}_{\mathbb{C}}\left(\mathfrak{g} / \mathfrak{t}, w^{-1} t\right)} \chi\left(\left(Y_{\sigma}\right)_{Q}, w^{-1} t\right)\right) \\
& =\sum_{Q \in \tilde{\mathcal{Q}}}(-1)^{\operatorname{codim} Q} \chi\left(M_{Q}, t\right) \\
& =\chi(M, t) .
\end{aligned}
$$

This completes the proof of Theorem 4.3.

\section{A. The equivariant index theorem for orbifolds.}

The equivariant version of Kawasaki's index theorem for orbifolds is due to M. Vergne [37]. A good reference is Chapter 14 in Duistermaat's book [15]; more information can be found in [26, Section 3]. We follow the conventions for the definition of a $G$-orbifold $M$ as given in [15].

The Kawasaki-Vergne formula expresses the equivariant index as an integral over connected components of a certain orbifold $\tilde{M}^{g}$. There is a natural surjection from $\tilde{M}^{g}$ onto the fixed point set $M^{g}$ of $g$, the latter however is not in general a sub-orbifold of $M$ :

Example A.1. Let $G=S^{1}$ act on $\mathbb{C}^{2}$ by $e^{i \phi} \cdot\left(z_{1}, z_{2}\right)=\left(e^{i \phi} z_{1}, e^{2 i \phi} z_{2}\right)$, and let $\mathbb{Z}_{2}$ act by $\left(z_{1}, z_{2}\right) \mapsto-\left(z_{1}, z_{2}\right)$. The $G$-action descends to $M=\mathbb{C}^{2} / \mathbb{Z}_{2}$. The fixed point set of $g=e^{i \pi} \in G$ is $M^{g}=\left\{\left(z_{1}, z_{2}\right) \mid z_{1} z_{2}=0\right\} / \mathbb{Z}_{2}$, which is not a sub-orbifold of $M$.

Given $m \in M^{g}$, let $(V, \Gamma, p)$ be a local orbifold chart around $m$. Thus $V$ is an open subset of $\mathbb{R}^{n}, \Gamma$ a finite group acting on $V$, and $p: V / \Gamma \rightarrow M$ a homeomorphism onto an open neighborhood of $m$. The action of $g$ on $V / \Gamma$ corresponds to some action on $V$, together with an automorphism $\phi$ of $\Gamma$ such that such that $\gamma \cdot g \cdot x=g \cdot \phi(\gamma) \cdot x$ for all $x \in V, \gamma \in \Gamma$. Let $\Gamma$ act on

$$
\tilde{V}^{g}:=\coprod_{\gamma \in \Gamma} V^{\gamma g} \times\{\gamma\}
$$


by $\gamma_{1} \cdot(x, \gamma)=\left(\gamma_{1} x, \gamma_{1} \gamma \phi\left(\gamma_{1}\right)^{-1}\right)$. The orbifold $\tilde{M}^{g}$ is obtained by gluing together the orbifolds $\widetilde{V / \Gamma}^{g}:=\tilde{V}^{g} / \Gamma$. Usually it has a number of connected components of different dimensions. The natural maps $\tilde{V}^{g} \rightarrow V$ descend to surjective maps $\widetilde{V / \Gamma}^{g} \rightarrow(V / \Gamma)^{g}$, which patch together to a surjective map $\tilde{M}^{g} \rightarrow M^{g}$. In our applications, the groups $\Gamma$ are abelian and the automorphism $\phi$ is trivial. In Example A.1, $\tilde{M}^{g}$ has two connected components:

$$
\tilde{M}^{g}=\left\{\left(z_{1}, z_{2}\right) \mid z_{2}=0\right\} / \mathbb{Z}_{2} \sqcup\left\{\left(z_{1}, z_{2}\right) \mid z_{1}=0\right\} / \mathbb{Z}_{2} .
$$

Suppose now that $(M, \omega, \Phi)$ is a Hamiltonian $G$-orbifold. Then all connected components of $\tilde{M}^{g}$ are symplectic manifolds, with symplectic form the pullback of $\omega$ under the map $\tilde{M}^{g} \rightarrow M$. If $M$ carries a $G$-equivariant prequantum line bundle $L \rightarrow M$ then its pull-back $\tilde{L} \rightarrow \tilde{M}^{g}$ is a pre-quantum line bundle for this symplectic structure. Local charts for $\tilde{L}$ are obtained from orbifold charts $(V, \Gamma, p)$ for $M$. In any such chart, $L$ is given by a $\Gamma$-equivariant pre-quantum line bundle $L_{V} \rightarrow V$, and the pull-back $L_{\tilde{V}^{g}}$ to $\tilde{V}^{g}$ is a $\Gamma$-equivariant line bundle, defining local charts for $\tilde{L}$.

At any point $(v, \gamma) \in \tilde{V}^{g}, \gamma g$ acts on the fiber over $(v, \gamma)$. The weight for this action depends only on the connected component $\widetilde{F}$ of $\tilde{M}^{g}$ containing $(v, \gamma)$, and will be denoted $\mu_{\widetilde{F}}(g) \in \mathrm{U}(1)$.

For any connected component $\widetilde{F}$, let $\mathrm{d}_{\widetilde{F}}$ denote its multiplicity, that is the number of elements in the orbifold isotropy group for a point in its smooth part. Let $\nu_{\widetilde{F}} \rightarrow \widetilde{F}$ be the normal bundle for the immersion $\widetilde{F} \rightarrow M$. In local orbifold charts $\tilde{V}^{g}$, it is given as the normal bundle $\nu_{\tilde{V}^{g}}$ of $\tilde{V}^{g}$ in $V \times \Gamma$. Again, its fiber at $(v, \gamma)$ carries an action of $\gamma g$. We let $\tilde{\mathcal{D}}_{\mathbb{C}}\left(\nu_{\widetilde{F}}, g\right) \in \Omega(\widetilde{F})$ be the differential form given in local charts by $\mathcal{D}_{\mathbb{C}}\left(\nu_{\tilde{V}^{g}}, \gamma g\right)$, using the definition of $\mathcal{D}_{\mathbb{C}}$ given in Section 2.3. Finally, we can state the fixed point theorem for this particular case:

Theorem A.2 (Vergne). Let $(M, \omega, \Phi)$ be a pre-quantized Hamiltonian $G$-orbifold. For any $g \in G$, the following fixed point formula holds:

$$
\chi(M, g)=\sum_{\widetilde{F} \subseteq \tilde{M}^{g}} \chi\left(\nu_{\widetilde{F}}, g\right)
$$

where

$$
\chi\left(\nu_{\widetilde{F}}, g\right)=\frac{1}{d_{\widetilde{F}}} \int_{\widetilde{F}} \frac{\operatorname{Td}(\widetilde{F}) \operatorname{Ch}(\tilde{L})}{\tilde{\mathcal{D}}_{\mathbb{C}}\left(\nu_{\widetilde{F}}, g\right)} \mu_{\widetilde{F}}(g) .
$$


We will also need a slightly more general version, expressing the index $\chi\left(M, g e^{\xi}\right)$ where $\xi$ is a sufficiently small element in the Lie algebra of the centralizer of $g$. Let $\operatorname{Ch}(\tilde{L}, \xi)$ and $\operatorname{Td}(\widetilde{F}, \xi)$ and $\tilde{\mathcal{D}}_{\mathbb{C}}\left(\nu_{\widetilde{F}}, g, \xi\right)$ denote the equivariant extensions, defined by replacing curvatures by equivariant curvatures in the definitions. (See e.g. the book [9]). Then Vergne in [37] proves the more general formula $\chi\left(M, g e^{\xi}\right)=\sum_{\widetilde{F} \subseteq \tilde{M}^{g}} \chi\left(\nu_{\widetilde{F}}, g, \xi\right)$ with

$$
\chi\left(\nu_{\widetilde{F}}, g, \xi\right)=\frac{1}{d_{\widetilde{F}}} \int_{\widetilde{F}} \frac{\operatorname{Td}(\widetilde{F}, \xi) \operatorname{Ch}(\tilde{L}, \xi)}{\tilde{\mathcal{D}}_{\mathbb{C}}\left(\nu_{\widetilde{F}}, g, \xi\right)} \mu_{\widetilde{F}}(g)
$$

\section{B. Symplectic Cutting.}

In this Section we explain the non-abelian version of Lerman's technique of symplectic cutting. In a nutshell, the method associates to any compact Hamiltonian $G$-space $(M, \omega, \Phi)$, and certain " $\Phi$-admissible" polytopes $Q \subset \mathfrak{t}^{*}$, a Hamiltonian $G$-orbifold $\left(M_{Q}, \omega_{Q}, \Phi_{Q}\right)$ with moment polytope $\Phi_{Q}\left(M_{Q}\right) \cap \mathfrak{t}_{+}^{*}=\Phi(M) \cap \mathfrak{t}_{+}^{*} \cap Q$. The space $M_{Q}$ is obtained from $G \cdot \Phi^{-1}\left(Q \cap \mathfrak{t}_{+}^{*}\right) \subset M$ by collapsing the boundary in a certain way.

\section{B.1. Labeled Polytopes.}

Let $T$ be a torus, with lattice $\Lambda \subset \mathfrak{t}$. A (rational) polyhedron $Q \subset \mathfrak{t}^{*}$ is a finite intersection of half spaces

$$
Q=\bigcap_{j=1}^{N}\left\{\mu \in \mathfrak{t}^{*} \mid\left\langle\mu, v_{j}\right\rangle \geq r_{j}\right\}
$$

where $N$ is the number of codimension 1 faces, $v_{j} \in \Lambda$ are non-zero lattice vectors and $r_{j} \in \mathbb{R}$. Compact polyhedra will be called polytopes. $Q$ is called simplicial if for all $\mu \in Q$, the vectors $v_{j}$ for which $\left\langle\mu, v_{j}\right\rangle=r_{j}$ are linearly independent. Following [25], we define a labeled polyhedron to be a polyhedron $Q \subset \mathfrak{t}^{*}$ with a choice of inward pointing normal vectors $v_{j} \in \Lambda$ for each codimension 1 face. From $Q$ and the labels $v_{j}$ one recovers the defining inequalities $\left\langle\mu, v_{j}\right\rangle \geq r_{j}$. We call a labeled polyhedron integral if all $r_{j} \in \mathbb{Z}$. Note this does not imply that the vertices of $Q$ are integral.

Associated to any codimension $k$ face $S$ of a simplicial labeled polyhedron $Q$ is a $k$-dimensional sub-torus $T_{S} \subset T$, with Lie algebra $\mathfrak{t}_{S}$ the space orthogonal to $S$. Letting $\left(v_{i_{1}}, \ldots, v_{i_{k}}\right)$ be the labels of codimension 1 faces

containing $S$, the map $\mathbb{R}^{k} \rightarrow \mathfrak{t}_{S}$ which takes the $j$ th standard basis vector 
to $v_{i_{j}}$ defines a covering $\left(S^{1}\right)^{k} \rightarrow T_{S}$, the kernel of which is isomorphic to the quotient of $\Lambda \cap \mathfrak{t}_{S}$ by the lattice generated by the vectors $v_{i_{j}}$.

\section{B.2. Non-abelian cutting.}

Let $(M, \omega, \Phi)$ be a connected Hamiltonian $G$-manifold. We denote by $\Psi: M \rightarrow \mathfrak{t}_{+}^{*}$ the composition of the moment map $\Phi$ with the quotient map $\mathfrak{g}^{*} \rightarrow \mathfrak{g}^{*} / \operatorname{Ad}(G)=\mathfrak{t}_{+}^{*}$. It is well-known that over $\Psi^{-1}\left(\operatorname{int}\left(\mathfrak{t}_{+}^{*}\right)\right)$, the map $\Psi$ is smooth and generates a $G$-equivariant Hamiltonian $T$-action. More generally, if $\sigma$ is an open face of $\mathfrak{t}_{+}^{*}$ and $Z\left(G_{\sigma}\right) \subseteq T$ the center of its centralizer, the composition of $\Psi$ with projection to $\mathfrak{z}\left(G_{\sigma}\right)^{*}$ is smooth near $\Psi^{-1}(\sigma)$, and generates a $G$-equivariant Hamiltonian $Z\left(G_{\sigma}\right)$-action.

Let $Q \subset \mathfrak{t}^{*}$ be a simplicial labeled polyhedron, with the property that $\Psi^{-1}(Q)$ is compact and connected. Suppose that

$$
\begin{aligned}
& \text { If } S \subset Q \text { and } \sigma \subset \mathfrak{t}_{+}^{*} \text { are open faces } \\
& \text { with } S \cap \sigma \cap \Psi(M) \neq \emptyset \text {, then } T_{S} \subseteq Z\left(G_{\sigma}\right) .
\end{aligned}
$$

It then follows that on a $G$-invariant neighborhood of $\Psi^{-1}(S)$, the composition of $\Psi$ with projection $p_{S}: \mathfrak{t}^{*} \rightarrow \mathfrak{t}_{S}^{*}$ generates a Hamiltonian $T_{S}$-action. The polyhedron $Q$ will be called $\Phi$-admissible if it has the property (43), and in addition satisfies

$$
\text { The action of } T_{S} \text { on } \Psi^{-1}(S) \text { is locally free. }
$$

Given a polyhedron $Q$ satisfying condition (43), condition (44) can be achieved by an arbitrarily small perturbation of the parameters $r_{j}$. Assuming (43), (44), choose a $G$-invariant neighborhood $U_{S}$ of $\Psi^{-1}(S)$ on which the action of $T_{S}$ is locally free.

Let $\left(S^{1}\right)^{k}$ act on $U_{S}$ by means of the covering, $\left(S^{1}\right)^{k} \rightarrow T_{S}$. As a moment map $\phi_{S}$ for this action we take the moment map $p_{S} \circ \Psi$ for the $T_{S^{-}}$action, shifted by $\nu_{S}=p_{S}(S)$ :

$$
\phi_{S}:=p_{S} \circ \Psi-\nu_{S}: U_{S} \rightarrow \mathfrak{t}_{S}^{*} \cong\left(\mathbb{R}^{k}\right)^{*} .
$$

The various torus actions and moment maps are compatible, in the sense that if $S_{1} \subset \overline{S_{2}}$, the restriction of $\phi_{S_{2}}$ to $U_{S_{1}} \cap U_{S_{2}}$ is a component of $\phi_{S_{1}}$. For any face $S$ consider the symplectic quotients

$$
\left(U_{S}\right)_{Q}:=\left(U_{S} \times \mathbb{C}^{k}\right) / /\left(S^{1}\right)^{k}
$$


under the diagonal action, using the standard action on $\mathbb{C}^{k}$. From the canonical isomorphism,

$$
\Psi^{-1}(Q) \cap U_{S}=\left(U_{S} \times \tilde{\mathbb{C}}^{k}\right) / /\left(S^{1}\right)^{k}
$$

where $\tilde{\mathbb{C}}$ is the symplectic manifold with boundary $\tilde{\mathbb{C}}=S^{1} \times \mathbb{R}_{+} \subset T^{*}\left(S^{1}\right)$, one sees that there is a canonical surjective map $\Psi^{-1}(Q) \cap U_{S} \rightarrow\left(U_{S}\right)_{Q}$, which is a symplectomorphism over $\Psi^{-1}(\operatorname{int}(Q))$.

One obtains a Hamiltonian $G$-orbifold $\left(M_{Q}, \omega_{Q}, \Phi_{Q}\right)$, called the cut space, by gluing the open subsets $\left(U_{S}\right)_{Q}$. The cut space is a union of $G$-invariant symplectic sub-orbifolds $M_{S}:=\Psi^{-1}(S) / T_{S}$, and there is a natural surjection $\Psi^{-1}(Q) \rightarrow M_{Q}$ which restricts to the quotient maps $\Psi^{-1}(S) \rightarrow M_{S}$. The normal bundle of $M_{S}$ in $M_{Q}$ is the associated orbifold bundle,

$$
\nu_{S}^{Q}=\Psi^{-1}(S) \times_{T_{S}}\left(\mathbb{C}^{k} / \Gamma_{S}\right) \rightarrow M_{S},
$$

where $\Gamma_{S}$ is the kernel of the homomorphism $\left(S^{1}\right)^{k} \rightarrow T_{S}$, and the action of $T_{S}$ is induced from the natural $\left(S^{1}\right)^{k}$-action on $\mathbb{C}^{k}$.

We now extend the cutting construction to $G$-equivariant vector bundles $E \rightarrow M$. Suppose that for all open faces $S \subset Q$ the $T_{S}$-action on $U_{S}$ lifts to a $G$-equivariant action of its cover $\left(S^{1}\right)^{k} \rightarrow T_{S}$, and that for faces $S_{1}, S_{2}$ with $S_{1} \subset \overline{S_{2}}$, these torus actions are compatible in the natural way. Define $\left(E \mid U_{S}\right)_{Q} \rightarrow\left(U_{S}\right)_{Q}$ by pulling $E$ back to $U_{S} \times \mathbb{C}^{k}$, restricting to the zero level set for the $\left(S^{1}\right)^{k}$-action, and taking the quotient. These local bundles glue together to give a $G$-equivariant orbifold bundle $E_{Q} \rightarrow M_{Q}$. Its restriction to $M_{S}$ is $E_{S}:=\left(E \mid \Psi^{-1}(S)\right) /\left(S^{1}\right)^{k}$.

We note that the cut $(T M)_{Q}$ of the tangent bundle $T M$ is not isomorphic to $T\left(M_{Q}\right)$. Indeed, for any face $S$ of $Q$,

$$
\left.(T M)_{Q}\right|_{M_{S}}=T\left(M_{S}\right) \oplus \mathbb{C}^{k} \quad \text { while }\left.\quad T\left(M_{Q}\right)\right|_{M_{S}}=T\left(M_{S}\right) \oplus \nu_{S}^{Q}
$$

Suppose $L \rightarrow M$ is a $G$-equivariant pre-quantum line bundle. The $T_{S}$-action on $U_{S}$ admits a $G$-equivariant pre-quantum lift (with respect to the moment map $\left.p_{S} \circ \Psi\right)$ to $L \mid U_{S}$. Since the moment map for $\left(S^{1}\right)^{k}$ is obtained by shift by $\nu_{S}$, the $\left(S^{1}\right)^{k}$-action admits a pre-quantum lift if and only if $\nu_{S} \in \mathfrak{t}_{S}^{*} \cong \mathbb{R}^{k}$ is contained in the lattice $\mathbb{Z}^{k}$. Clearly, this is the case if $Q$ is an integral labeled polyhedron. Since the trivial line bundle is pre-quantum for $\mathbb{C}^{k}$, the cut bundle $L_{Q} \rightarrow M_{Q}$ becomes then a $G$-equivariant pre-quantum bundle for the cut space. 


\section{B.3. The gluing formula.}

Let $(M, \omega, \Phi)$ be a compact, connected Hamiltonian $G$-manifold with prequantum line bundle $L \rightarrow M$. A $\Phi$-admissible labeled polyhedral subdivision of $\mathfrak{t}^{*}$ is a collection of $\Phi$-admissible labeled polyhedra $\mathcal{Q}=\{Q\}$ such that the collection covers $\mathfrak{t}^{*}$, the intersection of any two polyhedra is either empty or is a face of each, and the labels attached to a common codimension 1 face of any two polyhedra coincide up to sign. We call $\mathcal{Q}$ integral if all of the polytopes $Q \in \mathcal{Q}$ are integral labeled polyhedra. Let $\widetilde{\mathcal{Q}}$ be the collection of all closed faces of $Q \in \mathcal{Q}$; thus $\mathcal{Q}$ are the top-dimensional polyhedra in $\widetilde{\mathcal{Q}}$. To every $Q \in \widetilde{\mathcal{Q}}$ corresponds a pre-quantized Hamiltonian $G$-orbifold $M_{Q}$ which is a symplectic sub-orbifold in each $M_{Q^{\prime}}$, such that $Q$ is a closed face of $Q^{\prime} \in \widetilde{\mathcal{Q}}$. One has the following gluing formula [26] relating the $\operatorname{Spin}_{c^{-i n d i c e s}}$ of the cut spaces:

$$
\chi(M, g)=\sum_{Q \in \widetilde{\mathcal{Q}}}(-1)^{\operatorname{codim} Q} \chi\left(M_{Q}, g\right) .
$$

In this paper we need a refined version of (46), along ideas developed in [30]. Suppose $(M, \omega, \Phi)$ is a pre-quantized Hamiltonian $G$-manifold, and $S$ a $\Phi$-admissible integral labeled polyhedron. Consider the expression

$$
\chi_{S}(M, g)=\sum_{F \subseteq M^{g}} \mu_{F}(g) \int_{F \cap \Psi^{-1}(S)} \frac{\operatorname{Td}(F) \operatorname{Ch}(L)}{\mathcal{D}_{\mathbb{C}}\left(\nu_{F}, g\right)},
$$

where the representatives for the Todd class, Chern class and $\mathcal{D}_{\mathbb{C}}\left(\nu_{F}, g\right)$ are chosen in such a way that for all faces $R \subset S$, the pull-back to the submanifold $\Psi^{-1}(R)$ descends to a form on $M_{R}=\Psi^{-1}(R) / T_{R}$. Provided that the representatives satisfy this boundary condition, (47) is independent of their choice because the integral can be re-written as an integral over the cut space $F_{S} \subset\left(M^{t}\right)_{S} \subseteq M_{S}^{t}$ :

$$
\chi_{S}(M, g)=\sum_{F \subseteq M^{g}} \mu_{F}(g) \int_{F_{S}} \frac{\operatorname{Td}\left((T F)_{S}\right) \operatorname{Ch}\left(L_{S}\right)}{\mathcal{D}_{\mathbb{C}}\left(\left(\nu_{F}\right)_{S}, g\right)} .
$$

Suppose that $\mathcal{Q}=\{Q\}$ is a $\Phi$-admissible, integral, polyhedral subdivision, and also that all intersections $S \cap Q$ with $Q \in \mathcal{Q}$ are admissible. For any $Q \in \tilde{Q}$ let $\chi_{S}\left(M_{Q}, g\right)$ be defined by a formula similar to (47), by taking the integral in the Kawasaki-Vergne formula only over the part $\widetilde{F}_{S}$ mapping to $S$.

$$
\chi_{S}\left(M_{Q}, g\right)=\sum_{F \subseteq\left(M_{Q}\right)^{g}} \mu_{\widetilde{F}}(g) \int_{\widetilde{F}_{S}} \frac{\operatorname{Td}\left((T \widetilde{F})_{S}\right) \operatorname{Ch}\left(\left(\tilde{L}_{Q}\right)_{S}\right)}{\tilde{\mathcal{D}}_{\mathbb{C}}\left(\left(\nu_{\widetilde{F}}\right)_{S}, g\right)} .
$$


The following Proposition extends formula (34) in [30] to the equivariant case:

Proposition B.1. One has the gluing formula,

$$
\chi_{S}(M, g)=\sum_{Q \in \widetilde{\mathcal{Q}}}(-1)^{\operatorname{codim} Q} \chi_{S}\left(M_{Q}, g\right)
$$

Proof. The proof is an extension of the argument given in [30, p.465]. We may assume $g=t \in T$. Observe that all of the characteristic forms in (48) admit $T$-equivariant extensions. Hence we can write $\chi_{S}(M, t)=$ $\lim _{\xi \rightarrow 0} \chi_{S}(M, t, \xi)$ where

$$
\chi_{S}(M, t, \xi)=\sum_{F \subseteq M^{t}} \mu_{F}(t) \int_{F_{S}} \frac{\operatorname{Td}\left((T F)_{S}, \xi\right) \operatorname{Ch}\left(L_{S}, \xi\right)}{\mathcal{D}_{\mathbb{C}}\left(\left(\nu_{F}\right)_{S}, t, \xi\right)} .
$$

Let us apply the Berline-Vergne localization formula for orbifolds (cf. [26]) to this expression. Let $X$ be a fixed point manifold for the $T$-action on $\left(M^{t}\right)_{S}$. Letting $\Psi_{S}: M_{S} \rightarrow \mathfrak{t}_{+}^{*}$ be the map induced by $\Psi$, it follows that $\Psi_{S}(X)$ is a point. Let $R \subset S$ be the unique open face containing $\Psi_{S}(X)$, and let $F \subseteq M^{t}$ be the unique connected component with $X \subseteq F_{S}$. Recall that by (45) the restriction of $(T M)_{S}$ to $M_{R}$ is the tangent bundle of $M_{R}$ plus a trivial bundle. Similarly the restriction of $(T F)_{S}$ is the tangent bundle to $X$ plus a trivial bundle, and the restriction of $\left(\nu_{F}\right)_{S}$ is the normal bundle $\nu_{X}^{R}$ to $X$ in $M_{R}$. On the other hand, the normal bundle of $X$ in $F_{S}$ is the pull-back of the normal bundle $\nu_{R}^{S}$ of $M_{R}$ in $M_{S}$. We therefore obtain the formula

$$
\chi_{S}(M, t, \xi)=\sum_{X} \mu_{X}(t \exp \xi) \frac{1}{d_{X}} \int_{X} \frac{\operatorname{Td}(X) \operatorname{Ch}\left(L_{R}\right)}{\mathcal{D}_{\mathbb{C}}\left(\nu_{X}^{R}, t \exp \xi\right) \operatorname{Eul}\left(\nu_{R}^{S}, \xi\right)} .
$$

We obtain similar formulas for all of the cut spaces $M_{Q}$ :

$$
\chi_{S}\left(M_{Q}, t, \xi\right)=\sum_{X} \mu_{X}(t \exp \xi) \frac{1}{d_{X}} \int_{X} \frac{\operatorname{Td}(X) \operatorname{Ch}\left(\tilde{L}_{R}\right)}{\tilde{\mathcal{D}}_{\mathbb{C}}\left(\nu_{X}^{R}, t \exp \xi\right) \operatorname{Eul}\left(\nu_{R}^{S}, \xi\right)},
$$

where the sum is over all connected components $X$ of the $T$-fixed point set of $\widetilde{F}_{S}$, for all $F \subseteq M_{Q}^{t}$. If $X$ is a fixed point component for the $T$-action on $\left(M^{t}\right)_{S}$, then $\Psi(X)$ is contained in the interior of a unique top-dimensional polyhedron $Q \in \mathcal{Q}$. Hence, the fixed point contribution appears exactly once 
as a $T$-fixed point contribution of the sum $\sum_{Q \in \mathcal{Q}}(-1)^{\operatorname{codim} Q} \chi_{S}\left(M_{Q}, t, \xi\right)$. We must show that the remaining fixed point contributions cancel. These other integrals are over connected components $X$ of $T$-fixed point sets of $\widetilde{F}_{S}$, where $\widetilde{F} \subseteq \tilde{M}_{Q}^{t}$ is defined as in Appendix A. These fixed point components can be organized as follows. Consider the finite subset of points of the form $\left(\Psi_{Q}\right)_{S}(X) \in \mathfrak{A}$. Given any such point, there exists a unique open face $R$ of $S$ containing it. If $R=\operatorname{int}(S)$, then $X$ is simply one of the $T$ fixed point orbifolds for $M_{Q}$, and the cancellation of the corresponding fixed point contributions is just the gluing formula [26, Theorem 5.4]. In case $R$ is a proper face of $S$, the argument from [26] carries over without essential change, the reason being that the fixed point contributions look exactly like fixed point contributions for $M_{Q}$, except for the extra factor $\operatorname{Eul}\left(\nu_{R}^{S}, \xi\right)^{-1}$ which appears in all of these integrals.

\section{Fusion of group valued Hamiltonian $G$-spaces.}

In this appendix we collect some facts about fusion of Hamiltonian $G$-spaces with group-valued moment maps.

Theorem C.1 ([1, Theorem 6.1]). Let $G, H$ be compact Lie groups, and $\left(M, \omega,\left(\Phi_{1}, \Phi_{2}, \Psi\right)\right)$ a group valued Hamiltonian $G \times G \times H$-manifold. Let $M_{\text {fus }}$ be the same manifold with diagonal $G \times H$-action, $\Phi_{\text {fus }}=\Phi_{1} \Phi_{2}$, and $\omega_{\text {fus }}=\omega-\frac{1}{2} B\left(\Phi_{1}^{*} \theta, \Phi_{2}^{*} \bar{\theta}\right)$. Then $\left(M_{\text {fus }}, \omega_{\text {fus }},\left(\Phi_{\text {fus }}, \Psi\right)\right)$ is a group valued Hamiltonian $G \times H$-manifold.

The correction term $\frac{1}{2} B\left(\Phi_{1}^{*} \theta, \Phi_{2}^{*} \bar{\theta}\right)$ will be loosely referred to as the "fusion term". If $M=M_{1} \times M_{2}$ is a product of two $G \times H_{i}$-valued Hamiltonian spaces, we also write $M_{1} \circledast M_{2}:=\left(M_{1} \times M_{2}\right)_{\text {fus }}$.

Recall that if $G$ is a torus, a space with $G$-valued moment map is just a symplectic manifold with a multi-valued moment map in the usual sense. Fusion of such spaces changes the symplectic form, but not the volume:

Lemma C.2. Suppose $T$ is a torus, that $\left(M, \omega,\left(\Phi_{1}, \Phi_{2}\right)\right)$ a compact group valued Hamiltonian $T \times T$-space, and $\left(M_{\text {fus }}, \omega_{\text {fus }},\left(\Phi_{\text {fus }}\right)\right)$ is the group valued Hamiltonian T-space obtained by fusion. Then the symplectic volumes of $M$ and $M_{\text {fus }}$ are the same.

Proof. This is a special case of a result for non-abelian groups proved in [2]. In the abelian case, the following much simpler argument is available. Notice that $M$ with diagonal $T$-action has moment map $\Phi_{\text {fus }}=\Phi_{1} \Phi_{2}$ not only for 
the fusion form $\omega_{\text {fus }}$ but also for the original symplectic 2-form $\omega$. Suppose $t \in T$ is a (weakly) regular value of $\Phi_{\text {fus }}$, so that $\left(\Phi_{\text {fus }}\right)^{-1}(t)$ is a smooth submanifold and $M_{t}=\left(\Phi_{\text {fus }}\right)^{-1}(t) / T$ is an orbifold. Since the pull-back of the 2 -form $\frac{1}{2} B\left(\Phi_{1}^{*} \theta, \Phi_{2}^{*} \theta\right)$ to $\left(\Phi_{\text {fus }}\right)^{-1}(t)$ vanishes, the reduced symplectic forms are the same: $\omega_{t}=\left(\omega_{\text {fus }}\right)_{t}$. It follows that the two Duistermaat-Heckman measures $\mathfrak{m}=\frac{1}{n !}\left(\Phi_{\text {fus }}\right)_{*}\left(\left|\omega^{n}\right|\right)$ and $\mathfrak{m}_{\text {fus }}=\frac{1}{n !}\left(\Phi_{\text {fus }}\right)_{*}\left(\left|\omega_{\text {fus }}^{n}\right|\right)$ coincide. Since the symplectic volume is the integral of the Duistermaat-Heckman measure, the proof is complete.

For any group-valued Hamiltonian $G$-space, the 2 -form $\omega$ is nondegenerate on the tangent space at any point in the identity level set. The following Lemma shows that fusion does not change the isotopy class of this symplectic structure. Its proof relies on the notion of exponential of a Hamiltonian space [1]: Let $\varpi \in \Omega^{2}(\mathfrak{g})$ be the image of $\exp ^{*} \eta$ under the homotopy operator $\Omega^{\star}(\mathfrak{g}) \rightarrow \Omega^{\star-1}(\mathfrak{g})$. Then if $\left(M, \omega_{0}, \Phi_{0}\right)$ is a Hamiltonian $G$-space in the usual sense, with $\Phi_{0}(M)$ contained in a sufficiently small neighborhood of 0 , then $(M, \omega, \Phi)$ with $\omega=\omega_{0}+\Phi_{0}^{*} \varpi$ and $\Phi=\exp \left(\Phi_{0}\right)$ is a group valued Hamiltonian $G$-space. Conversely, if $(M, \omega, \Phi)$ is a groupvalued Hamiltonian $G$-space, any small neighborhood of $\Phi^{-1}(e)$ is obtained in this way. The 2 -form $\varpi$ vanishes at 0 , so that $\omega_{m}=\left(\omega_{0}\right)_{m}$ for points in the zero level set.

Lemma C.3. Let $\left(M, \omega,\left(\Phi_{1}, \Phi_{2}, \Psi\right)\right)$ be a group valued Hamiltonian $G \times$ $G \times H$-space, and $\left(M_{\text {fus }}, \omega_{\text {fus }},\left(\Phi_{\text {fus }}, \Psi\right)\right)$ its fusion. Let $m \in M$ be a point in the identity level set of $\left(\Phi_{1}, \Phi_{2}, \Psi\right)$. The symplectic 2 -forms $\left.\omega\right|_{m}$ and $\left.\omega_{\text {fus }}\right|_{m}$ on $T_{m} M$ are isotopic through a path of symplectic forms, invariant under the stabilizer group $(G \times H)_{m}$.

Proof. We may assume that $M$ is the exponential of a Hamiltonian $G \times G \times H$ space $\left(M, \omega_{0},\left(\Phi_{0,1}, \Phi_{0,2}, \Psi_{0}\right)\right)$. Rescaling by $s>0$, we obtain a family of Hamiltonian spaces $\left(M, s \omega_{0},\left(s \Phi_{0,1}, s \Phi_{0,2}, s \Psi_{0}\right)\right)$, together with their exponentials. Let $\omega_{\text {fus }}^{s}$ be the corresponding fusion forms. We claim that $\left.s^{-1} \omega_{\text {fus }}^{s}\right|_{m}$ give the required isotopy of symplectic forms. Indeed, each $\left.\omega_{\text {fus }}^{s}\right|_{m}$ is symplectic, and for $s \rightarrow 0$,

$$
\left.\omega_{\text {fus }}^{s}\right|_{m}=\left.s \omega_{0}\right|_{m}-\left.\frac{1}{2} B\left(\exp \left(s \Phi_{0,1}\right)^{*} \theta, \exp \left(s \Phi_{0,2}\right)^{*} \bar{\theta}\right)\right|_{m}=\left.s \omega_{0}\right|_{m}+O\left(s^{2}\right)
$$

showing that $\left.\lim _{s \rightarrow 0} s^{-1} \omega_{\text {fus }}^{s}\right|_{m}=\left.\omega_{0}\right|_{m}$. 


\section{References.}

[1] A. Alekseev, A. Malkin, and E. Meinrenken, Lie group valued moment maps, J. Differential Geom., 48(3) (1998), 445-495.

[2] A. Alekseev, E. Meinrenken, and C. Woodward, Duistermaat-Heckman distributions for Lie group valued moment maps, preprint, 1999.

[3] M.F. Atiyah, The geometry and physics of knots, Cambridge University Press, Cambridge, 1990.

[4] M.F. Atiyah and R. Bott, A Lefschetz fixed point formula for elliptic complexes I, Ann. of Math., 86(2) (1967), 374-407.

[5] M.F. Atiyah and R. Bott, A Lefschetz fixed point formula for elliptic complexes II, Applications, Ann. of Math., 88(2) (1968), 451-491.

[6] M.F. Atiyah and G.B. Segal, The index of elliptic operators, II, Ann. of Math., 87(2) (1968), 531-545.

[7] M.F. Atiyah and I.M. Singer, The index of elliptic operators I, Ann. of Math., 87(2) (1968), 484-530.

[8] A. Beauville, Conformal blocks, fusion rules and the Verlinde formula, in 'Proceedings of the Hirzebruch 65 Conference on Algebraic Geometry' (Ramat Gan, 1993), 9, Israel Math. Conf. Proc., 75-96, Bar-Ilan Univ., Ramat Gan, 1996.

[9] N. Berline, E. Getzler, and M. Vergne, Heat Kernels and Dirac Operators, Grundlehren der mathematischen Wissenschaften, 298, SpringerVerlag, Berlin-Heidelberg-New York, 1992.

[10] J.M. Bismut and F. Labourie, Symplectic geometry and the Verlinde formulas, in 'Surveys in differential geometry: differential geometry inspired by string theory', 97-311, Int. Press, Boston, MA, 1999.

[11] M. Blau and G. Thompson, Equivariant Kähler geometry and localization in the $G / G$ model, Nuclear Phys. B, 439(1-2) (1995), 367-394.

[12] T. Bröcker and T. tom Dieck, Representations of Compact Lie Groups, Graduate Texts in Mathematics, 98, Springer-Verlag, BerlinHeidelberg-New York, 1985. 
[13] S. Chang, Fixed point formula and loop group actions, math. AG/9812148.

[14] S.K. Donaldson, Boundary value problems for Yang-Mills fields, J. Geom. Phys., 8 (1992), 89-122.

[15] J.J. Duistermaat, The Heat Kernel Lefschetz Fixed Point Formula for the Spin-c Dirac Operator, Progress in Nonlinear Differential Equations and Their Applications, 18, Birkhäuser, Boston, 1996.

[16] D. Freed, The Verlinde algebra is twisted equivariant K-theory, Technical report, 2000.

[17] D.S. Freed, Classical Chern-Simons theory, I, Adv. Math., 113(2) (1995), 237-303.

[18] A. Gerasimov, Localization in $G W Z W$ and Verlinde formula, preprint, Uppsala, 1993, hep-th/9305090.

[19] V. Guillemin and E. Prato, Heckman, Kostant, and Steinberg formulas for symplectic manifolds, Adv. in Math., 82(2) (1990), 160-179.

[20] V. Guillemin and S. Sternberg, Symplectic Techniques in Physics, Cambridge Univ. Press, Cambridge, 1990.

[21] L.C. Jeffrey and F.C. Kirwan, Intersection pairings in moduli spaces of holomorphic bundles on a Riemann surface, Electron. Res. Announc. Amer. Math. Soc., 1(2) (1995), 57-71 (electronic).

[22] F.C. Kirwan, Convexity properties of the moment mapping, III, Invent. Math., 77 (1984), 547-552.

[23] B. Kostant, Quantization and unitary representations, in C. T. Taam, editor, Lectures in Modern Analysis and Applications III, Lecture Notes in Mathematics, 170, 87-208, Washington, D.C., 1970, SpringerVerlag, Berlin-Heidelberg-New York.

[24] H.B. Lawson, Jr. and M.-L. Michelson, Spin Geometry, Princeton Mathematical Series, 38, Princeton Univ. Press, Princeton, 1989.

[25] E. Lerman and S. Tolman, Hamiltonian torus actions on symplectic orbifolds and toric varieties, Trans. Amer. Math. Soc., 349(10) (1997), 4201-4230. 
[26] E. Meinrenken, Symplectic surgery and the Spin ${ }^{\mathrm{c}}$-Dirac operator, Adv. in Math., 134 (1998), 240-277.

[27] E. Meinrenken and R. Sjamaar, Singular reduction and quantization, Topology, 38 (1999), 699-763.

[28] E. Meinrenken and C. Woodward, Canonical bundles for Hamiltonian loop group actions, Pacific J. of Math., to appear.

[29] E. Meinrenken and C. Woodward, Cobordism for Hamiltonian loop group actions and flat connections on the punctured two-sphere, Math. Z., 231(1) (1999), 133-168.

[30] E. Meinrenken and C. Woodward, Hamiltonian loop group actions and Verlinde factorization, J. Differential Geom., 50 (1999), 417-470.

[31] A. Pressley and G. Segal, Loop groups, Oxford University Press, Oxford, 1988.

[32] R. Sjamaar and E. Lerman, Stratified symplectic spaces and reduction, Ann. of Math., 134(2) (1991), 375-422.

[33] A. Szenes, The combinatorics of the Verlinde formulas, in 'Vector bundles in algebraic geometry' (Durham, 1993), London Math. Soc. Lecture Note Ser., 208, 241-253, Cambridge Univ. Press, 1995.

[34] C. Teleman, The quantization conjecture revisited, Ann. of Math. (2), $\mathbf{1 5 2}(\mathbf{1})(2000), 1-43$.

[35] V. Toledano Laredo, Positive energy representations of the loop groups of non-simply connected groups, Comm. Math. Phys., 207 (1999), 307339 .

[36] A. Tsuchiya, K. Ueno, and Y. Yamada, Conformal field theory on universal family of stable curves with gauge symmetries, in 'Integrable systems in quantum field theory and statistical mechanics', Adv. Stud. Pure Math., 19, 459-566, Academic Press, 1989.

[37] M. Vergne, Equivariant index formula for orbifolds, Duke Math. J., 82 (1996), 637-652. 
[38] E. Verlinde, Fusion rules and modular transformations in $2 d$ conformal field theory, Nuclear Phys. B, 300 (1988), 360-376.

\author{
Institute for Theoretical Physics \\ UPPSALA UNIVERSITY \\ Box 803 \\ S-75108 UPPSALA \\ SWEDEN \\ E-mail address: alekseev@teorfys.uu.se \\ UNIVERSITY OF TORONTO \\ Department of Mathematics \\ 100 St George Street, Toronto \\ Ontario M5R3G3, Canada \\ E-mail address: mein@math.toronto.edu \\ Mathematics-Hill Center \\ RUTGERS UNIVERSITY \\ 110 Frelinghuysen RoAD \\ PiscataWAY NJ 08854-8019 \\ E-mail address: ctw@math.rutgers.edu
}

